\title{
Theoretical full-field analysis of dissimilar isotropic composite annular wedges under anti-plane deformations
}

\author{
Ru-Li Lin ${ }^{\text {a }}$, Chien-Ching Ma ${ }^{\text {b,* }}$ \\ a Department of Mechanical Engineering, Southern Taiwan University of Technology, Tainan County, \\ Taiwan 71008, Taiwan, ROC \\ ${ }^{\mathrm{b}}$ Department of Mechanical Engineering, National Taiwan University, No. 1 Roosevelt Rd, Sec. 4, Taipei 10617, Taiwan, ROC
}

Received 7 May 2004; accepted 7 May 2004

Available online 17 June 2004

\begin{abstract}
By using the Mellin transform technique in conjunction with the image method, the two-dimensional full-field solutions of dissimilar isotropic composite annular wedges subjected to anti-plane concentrated forces and screw dislocations are presented in explicit forms. The composite wedges consist of two materials that have equal apex angle and are bonded together along an interface. The explicit full-field solutions are presented in series forms for combinations of traction and displacement boundary conditions. For the special case of composite sharp wedges with finite radius or infinite extent, the solutions with functional forms are obtained and only consist of simple trigonometric functions. Explicit solutions of the stress intensity factors are obtained for a semi-infinite interface crack and a circular composite disk with an interface crack. With the aid of the Peach-Koehler equation, the explicit forms of the image forces exerted on screw dislocations are easily derived from the full-field solutions of stresses. Numerical results of full-field stress distributions and image forces exerted on screw dislocations are presented and discussed in detail.
\end{abstract}

(c) 2004 Elsevier Ltd. All rights reserved.

Keywords: Image method; Composite annular wedge; Mellin transform; Interface crack; Screw dislocation

\section{Introduction}

The stress analysis for the wedge problem with infinite length has been investigated by many authors. Some authors devoted efforts on deriving the full-field stress distribution in the wedge and others investigate the stress singularities near the apex of the wedge. The isotropic wedge problem was first considered by Tranter (1948) by using the Mellin transform in conjunction with the Airy stress function representation of plane elasticity. Williams (1952) obtained the solution of dissimilar materials with a semi-infinite interface crack and observed the stress oscillation near the crack tip. Chou (1965) investigated the screw dislocation

\footnotetext{
${ }^{*}$ Corresponding author. Tel.: +886-2-23659996; fax: +886-2-23631755.

E-mail address: ccma@ntu.edu.tw (C.-C. Ma).
} 
near a shape wedge boundary by means of the conformal mapping technique. In his study, the method of image for anti-plane single wedge and image force on screw dislocation was discussed in detail. Bogy (1971, 1972) used complex function representation of the generalize Mellin transform to obtain the solution of the plane problem of dissimilar wedge and single wedge with infinite extent. Ma and Hour (1989) investigated the problem of dissimilar anisotropic wedge subjected to anti-plane deformation and discussed the stress singularity near the apex of wedge. Ting $(1984,1985)$ discussed the paradox which existed in the elementary solution of an elastic wedge. Zhang et al. (1995) studied the problem for the interaction of an edge dislocation with a wedge crack. Kargarnovin et al. (1997) solved the problem of an isotropic wedge with finite radius subjected to anti-plane surface traction. The solution of anisotropic single wedge was obtained by Shahani (1999). However, both of the solutions were represented with an infinite series form. Kargarnovin (2000) studied the dissimilar finite wedge under anti-plane deformation and obtained only the near-tip field solution. Wang et al. (1986) investigated the stress intensity factor for the rigid line inclusion under antiplane shear loading. He and Hutchinson (1989a,b) analyzed several problems which provided insight and quantitative information on the role an interface between dissimilar elastic materials plays when approached by a crack. The condition that a crack impinging on an interface will pass through the interface or be deflected into the interface was discussed in detail.

For engineering applications, layer and wedge configurations are two problems that are commonly analyzed in the literature. The use of the image method in solving two-dimensional anti-plane problems is well-known. The anti-plane full-field solution of a single layer can be obtained by using an infinite array of image singularities to account for the boundary conditions of the two free or fixed surfaces. The results are identical to the work by using the method of Fourier transform in conjunction with series expansion. Chou (1966) used the technique of image method to construct the full-field solution of three phase lamellar structure subjected to a screw dislocation. The anisotropic case was obtained by Lin and Chou (1975). Chu (1982) used the conformal mapping technique to construct the closed-form solution of two phase isotropic thin film subjected to screw dislocation. The image method plays the essential role in these works. The method of image has been successfully extended to solve the problem of multilayered media with anti-plane shear deformation. By using a linear coordinate transformation and the Fourier transform technique, an effective analytical methodology was developed by Lin and Ma (2000) to obtain explicit analytical solutions for an anisotropic multilayered medium with $n$ layers subjected to an anti-plane loading or a screw dislocation in an arbitrary layer. However, the image method for wedge problem under anti-plane deformation is restricted for special apex angles (Chou, 1965).

In this study, the finite annular dissimilar composite wedge with equal apex angle subjected to anti-plane concentrated loadings and screw dislocations is investigated by analytical methods. The boundary conditions prescribed on radial edges, either tractions or displacements, are discussed in detail. In each problem, different boundary conditions prescribed on the circular segments are presented with the aid of the image method. The analytical solutions of composite sharp wedges with infinite length along the radial direction are first solved by a straightforward application of the Mellin transform, and the solutions are expressed in simple explicit functional forms. Based on the image method, the full-field solutions for composite sharp wedges with a finite radius are also presented in explicit functional forms. The analytical solution of the stress intensity factor of the circular composite disk with an interface crack is also obtained. In order to solve the finite annular dissimilar wedge problem, the image method is used to satisfy the boundary conditions on two circular segments based on the available solutions with functional forms of the infinite wedge problem. Base on the complete analytical solutions of stress fields for the wedge problem, the image forces exerted on screw dislocations are given in explicit forms with the aid of the Peach-Koehler equation. Numerical calculations of stress distributions are provided for traction or displacement boundary conditions. The full-field stress distributions and image forces exerted on screw dislocations for composite sharp wedge with finite radius and finite annular dissimilar composite wedge are studied in detail from numerical investigations. 


\section{Basic equations and general solutions}

Consider an isotropic composite sharp wedge with infinite length along the radial direction and with an apex angle $\beta(0 \leqslant \beta \leqslant 2 \pi)$ as shown in Fig. 1 . Let 1,2 denote the open two-dimensional regions which occupies the same apex angle $\beta / 2$. The composite wedge is perfectly bonded together along a common edge. For the anti-plane shear deformation, the non-vanishing displacement component is along the $z$-axis, $w(r, \theta)$, which is a function of in-plane coordinates $r$ and $\theta$. In the absence of body forces, the equilibrium equation for a homogeneous isotropic material in terms of displacement is given by

$$
\frac{\partial^{2} w}{\partial r^{2}}+\frac{1}{r} \frac{\partial w}{\partial r}+\frac{1}{r^{2}} \frac{\partial^{2} w}{\partial \theta^{2}}=0
$$

The non-vanishing shear stresses are

$$
\tau_{r z}(r, \theta)=\mu \frac{\partial w}{\partial r}, \quad \tau_{\theta z}(r, \theta)=\frac{\mu}{r} \frac{\partial w}{\partial \theta}
$$

where $\mu$ denotes the shear modulus of an isotropic material. In addition, we shall require the stress fields to satisfy the regularity conditions

$$
\tau_{r z}, \tau_{\theta z}=\mathrm{O}\left(r^{-1+\delta}\right) \quad \text { as } r \rightarrow \infty \text { for } \delta>0
$$

Mellin transform method is convenient for solving the problems in polar coordinate. Let the Mellin transform of a function $f(r)$ be denoted by $\hat{f}(s)$, then

$$
\hat{f}(s)=M[f(r), s]=\int_{0}^{\infty} f(r) r^{s-1} \mathrm{~d} r, \quad f(r)=M^{-1}[\hat{f}(s), r]=\frac{1}{2 \pi i} \int_{c-\mathrm{i} \infty}^{c+\mathrm{i} \infty} \hat{f}(s) r^{-s} \mathrm{~d} s
$$

where $s$ is a complex transform parameter. The Mellin transform of $w(r, \theta), r \tau_{r z}(r, \theta)$ and $r \tau_{\theta z}(r, \theta)$ in the transform domain are given by

$$
\begin{aligned}
& \hat{w}(s, \theta)=\int_{0}^{\infty} w(r, \theta) r^{s-1} \mathrm{~d} r \\
& \hat{\tau}_{r z}(s, \theta)=\int_{0}^{\infty} \tau_{r z}(r, \theta) r^{s} \mathrm{~d} r \\
& \hat{\tau}_{\theta z}(s, \theta)=\int_{0}^{\infty} \tau_{\theta z}(r, \theta) r^{s} \mathrm{~d} r
\end{aligned}
$$

By use of the inversion theorem for the Mellin transform, the stresses and displacement components are given by

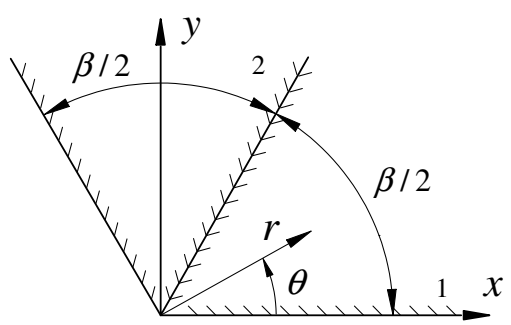

Fig. 1. The geometry configuration and coordinate of a composite sharp wedge with infinite length and equal apex angle $\beta / 2$. 


$$
\begin{aligned}
& w(r, \theta)=\frac{1}{2 \pi i} \int_{\rho-\mathrm{i} \infty}^{\rho+\mathrm{i} \infty} \hat{w}(s, \theta) r^{-s} \mathrm{~d} s \\
& \tau_{r z}(r, \theta)=\frac{1}{2 \pi i} \int_{\rho-\mathrm{i} \infty}^{\rho+\mathrm{i} \infty} \hat{\tau}_{r z}(s, \theta) r^{-s-1} \mathrm{~d} s \\
& \tau_{\theta z}(r, \theta)=\frac{1}{2 \pi i} \int_{\rho-\mathrm{i} \infty}^{\rho+\mathrm{i} \infty} \hat{\tau}_{\theta z}(s, \theta) r^{-s-1} \mathrm{~d} s
\end{aligned}
$$

Because of condition (2), the path of integration in the complex line integrals $\operatorname{Re}(s)=\rho$ in (4a)-(4c) must lie within a common strip of regularity of their intergrands, the choice of $\rho$ is taken to be

$$
\rho=-\varepsilon \quad 0<\varepsilon<\left(\left|\operatorname{Re}\left(s_{1}\right)\right|\right)
$$

where $s_{1}$ denotes the location of the pole in the open strip $-1<\operatorname{Re}(s)<0$ with the largest real part and $\operatorname{Re}$ denotes the real part of the complex argument.

Applying the Mellin transform (3a) to (1) yields an ordinary differential equation for $\hat{w}(s, \theta)$, the general solution of this ordinary differential equation is readily known to be

$$
\hat{w}(s, \theta)=c_{1} \sin (s \theta)+c_{2} \cos (s \theta)
$$

where $c_{1}$ and $c_{2}$ can be determined from the boundary conditions. The general solutions of stress components in the transform domain are

$$
\begin{aligned}
& \hat{\tau}_{r z}(s, \theta)=-\mu s\left(c_{1} \sin (s \theta)+c_{2} \cos (s \theta)\right) \\
& \hat{\tau}_{\theta z}(s, \theta)=\mu s\left(c_{1} \cos (s \theta)-c_{2} \sin (s \theta)\right)
\end{aligned}
$$

\section{Green's function of infinite composite wedge}

\subsection{Free-free boundary condition}

Consider a composite sharp wedge with infinite length along the radial direction and possess the apex angle $\beta$ subjected to a concentrated loading $f_{z}$ located at $(r, \theta)=(d, \alpha)$ in material 1 as shown in Fig. 2. Perfect bonding along the interface $\theta=\beta / 2$ is ensured by the stress and displacement continuity conditions, and the traction free boundary conditions on the two radial edges are considered first in this section. The region of the wedge is divided into three parts along the apex of wedge and the location of concentrated loading as shown in Fig. 2. The general solutions for material 1 are expressed as

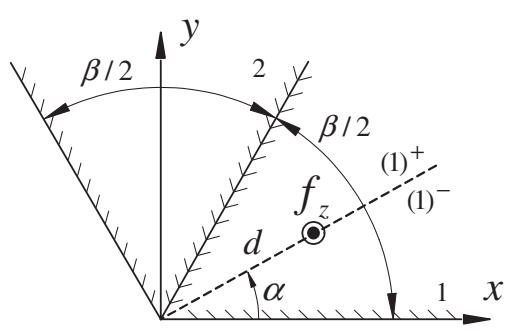

Fig. 2. A composite sharp wedge with infinite length subjected to a concentrated force located at $r=d$ and $\theta=\alpha$. 


$$
\begin{array}{ll}
\hat{w}^{(1)^{-}}(s, \theta)=c_{1}^{-} \sin (s \theta)+c_{2}^{-} \cos (s \theta) & \text { for } 0 \leqslant \theta<\alpha \\
\hat{w}^{(1)^{+}}(s, \theta)=c_{1}^{+} \sin (s \theta)+c_{2}^{+} \cos (s \theta) & \text { for } \alpha<\theta \leqslant \beta / 2
\end{array}
$$

For material 2, the general solution is

$$
\hat{w}^{(2)}(s, \theta)=d_{1} \sin (s \theta)+d_{2} \cos (s \theta) \text { for } \beta / 2 \leqslant \theta \leqslant \beta
$$

The associated traction free boundary conditions on the two radial edges are given by

$$
\left.\hat{\tau}_{\theta z}^{(1)^{-}}\right|_{\theta=0}=0,\left.\quad \hat{\tau}_{\theta z}^{(2)}\right|_{\theta=\beta}=0
$$

The jump conditions along $\theta=\alpha$ on material 1 are expressed as

$$
\left.\hat{\tau}_{\theta z}^{(1)^{+}}\right|_{\theta=\alpha^{+}}-\left.\hat{\tau}_{\theta z}^{(1)^{-}}\right|_{\theta=\alpha^{-}}=-f_{z} \mathrm{~d}^{s},\left.\quad w^{(1)^{+}}\right|_{\theta=\alpha^{+}}-\left.w^{(1)^{-}}\right|_{\theta=\alpha^{-}}=0
$$

The continuity conditions along the interface $\theta=\beta / 2$ are

$$
w^{(1)^{+}}(r, \beta / 2)=w^{(2)}(r, \beta / 2), \quad \tau_{\theta z}^{(1)^{+}}(r, \beta / 2)=\tau_{\theta z}^{(2)}(r, \beta / 2)
$$

From Eqs. (7a), (7b) and (8) with the aid of conditions (9)-(11), the complete solutions in the transform domain for materials 1 and 2 are

$$
\begin{aligned}
& \hat{w}^{(1)}=\frac{f_{z} d^{s}}{2 \mu_{1} s \sin (s \beta)}[\cos (s(\beta-\theta+\alpha))+\cos (s(\beta-\theta-\alpha))+k \cos (s(\theta-\alpha))+k \cos (s(\theta+\alpha))] \\
& \hat{\tau}_{r z}^{(1)}=\frac{-f_{z} d^{s}}{2 \sin (s \beta)}[\cos (s(\beta-\theta+\alpha))+\cos (s(\beta-\theta-\alpha))+k \cos (s(\theta-\alpha))+k \cos (s(\theta+\alpha))] \\
& \hat{\tau}_{\theta z}^{(1)}=\frac{f_{z} d^{s}}{2 \sin (s \beta)}[\sin (s(\beta-\theta+\alpha))+\sin (s(\beta-\theta-\alpha))-k \sin (s(\theta-\alpha))-k \sin (s(\theta+\alpha))] \\
& \hat{w}^{(2)}=\frac{f_{z} d^{s}}{\left(\mu_{1}+\mu_{2}\right) s \sin (s \beta)}[\cos (s(\beta-\theta+\alpha))+\cos (s(\beta-\theta-\alpha))] \\
& \hat{\tau}_{r z}^{(2)}=\frac{-\mu_{2} d^{s} f_{z}}{\left(\mu_{1}+\mu_{2}\right) \sin (s \beta)}[\cos (s(\beta-\theta+\alpha))+\cos (s(\beta-\theta-\alpha))] \\
& \hat{\tau}_{\theta z}^{(2)}=\frac{\mu_{2} d^{s} f_{z}}{\left(\mu_{1}+\mu_{2}\right) \sin (s \beta)}[\sin (s(\beta-\theta+\alpha))+\sin (s(\beta-\theta-\alpha))]
\end{aligned}
$$

where $k=\left(\mu_{1}-\mu_{2}\right) /\left(\mu_{1}+\mu_{2}\right)$. Rewrite the apex angle $\beta$ in the form $\beta=\pi / n, n$ is a real positive number and $1 / 2 \leqslant n<\infty$. When $n=1 / 2$, we have $\beta=2 \pi$, and this corresponds to the bimaterial interface crack problem. The useful formulations of inverse Mellin transform are summarized as follows:

$$
\begin{aligned}
& M^{-1}\left[\frac{\sin (s \phi)}{\sin \left(s \frac{\pi}{n}\right)}\right]=\frac{n}{\pi} \frac{r^{n} \sin (n \phi)}{1+2 r^{n} \cos (n \phi)+r^{2 n}} \quad 0<\operatorname{Re}[s]<1, \quad 0<\phi<\pi / n, \quad n \geqslant 1 / 2 \\
& M^{-1}\left[\frac{\cos (s \phi)}{\sin \left(s \frac{\pi}{n}\right)}\right]=\frac{n}{\pi} \frac{1+r^{n} \cos (n \phi)}{1+2 r^{n} \cos (n \phi)+r^{2 n}}
\end{aligned}
$$

The complete solutions of displacement and stresses for material 1 subjected to a concentrated loading are obtained with the formulations given in Eqs. (12a) and (13) as follows 


$$
\begin{aligned}
& w^{(1)}=\frac{f_{z}}{4 \pi \mu_{1}}\left[\Omega^{-}\left((r / d)^{n}, n(\theta-\alpha)\right)+\Omega^{-}\left((r / d)^{n}, n(\theta+\alpha)\right)+k \Omega^{+}\left((r / d)^{n}, n(\theta-\alpha)\right)+k \Omega^{+}\left((r / d)^{n}, n(\theta+\alpha)\right)\right] \\
& \tau_{r z}^{(1)}=\frac{n f_{z}}{2 \pi r}\left[\Gamma^{-}\left((r / d)^{n}, n(\theta-\alpha)\right)+\Gamma^{-}\left((r / d)^{n}, n(\theta+\alpha)\right)+k \Gamma^{+}\left((r / d)^{n}, n(\theta-\alpha)\right)+k \Gamma^{+}\left((r / d)^{n}, n(\theta+\alpha)\right)\right] \\
& \tau_{\theta z}^{(1)}=\frac{n f_{z}}{2 \pi r}\left[\Theta^{-}\left((r / d)^{n}, n(\theta-\alpha)\right)+\Theta^{-}\left((r / d)^{n}, n(\theta+\alpha)\right)-k \Theta^{+}\left((r / d)^{n}, n(\theta-\alpha)\right)-k \Theta^{+}\left((r / d)^{n}, n(\theta+\alpha)\right)\right]
\end{aligned}
$$

For material 2, the full-field solutions are

$$
\begin{aligned}
w^{(2)} & =\frac{f_{z}}{2 \pi\left(\mu_{1}+\mu_{2}\right)}\left[\Omega^{-}\left((r / d)^{n}, n(\theta-\alpha)\right)+\Omega^{-}\left((r / d)^{n}, n(\theta+\alpha)\right)\right] \\
\tau_{r z}^{(2)} & =\frac{n \mu_{2} f_{z}}{\pi\left(\mu_{1}+\mu_{2}\right) r}\left[\Gamma^{-}\left((r / d)^{n}, n(\theta-\alpha)\right)+\Gamma^{-}\left((r / d)^{n}, n(\theta+\alpha)\right)\right] \\
\tau_{\theta z}^{(2)} & =\frac{n \mu_{2} f_{z}}{\pi\left(\mu_{1}+\mu_{2}\right) r}\left[\Theta^{-}\left((r / d)^{n}, n(\theta-\alpha)\right)+\Theta^{-}\left((r / d)^{n}, n(\theta+\alpha)\right)\right]
\end{aligned}
$$

where

$$
\begin{aligned}
& \Omega^{ \pm}(R, \Phi)=\ln \left(1 \pm 2 R \cos \Phi+R^{2}\right), \quad \Gamma^{ \pm}(R, \Phi)=\frac{R^{2} \pm R \cos \Phi}{1 \pm 2 R \cos \Phi+R^{2}} \\
& \Theta^{ \pm}(R, \Phi)=\frac{R \sin \Phi}{1 \pm 2 R \cos \Phi+R^{2}}
\end{aligned}
$$

The full-field solutions of displacement and stresses for a screw dislocation with Burger's vector $b_{z}$ located at $(r, \theta)=(d, \alpha)$ can be obtained by the similar procedure and the results are

$$
\begin{aligned}
& w^{(1)}=\frac{-b_{z}}{2 \pi}\left[\Psi^{-}\left((r / d)^{n}, n(\theta-\alpha)\right)-\Psi^{-}\left((r / d)^{n}, n(\theta+\alpha)\right)+k \Psi^{+}\left((r / d)^{n}, n(\theta-\alpha)\right)-k \Psi^{+}\left((r / d)^{n}, n(\theta+\alpha)\right)\right] \\
& \tau_{r z}^{(1)}=\frac{n \mu_{1} b_{z}}{2 \pi r}\left[\Theta^{-}\left((r / d)^{n}, n(\theta-\alpha)\right)-\Theta^{-}\left((r / d)^{n}, n(\theta+\alpha)\right)-k \Theta^{+}\left((r / d)^{n}, n(\theta-\alpha)\right)+k \Theta^{+}\left((r / d)^{n}, n(\theta+\alpha)\right)\right] \\
& \tau_{\theta z}^{(1)}=\frac{-n \mu_{1} b_{z}}{2 \pi r}\left[\Gamma^{-}\left((r / d)^{n}, n(\theta-\alpha)\right)-\Gamma^{-}\left((r / d)^{n}, n(\theta+\alpha)\right)+k \Gamma^{+}\left((r / d)^{n}, n(\theta-\alpha)\right)-k \Gamma^{+}\left((r / d)^{n}, n(\theta+\alpha)\right)\right] \\
& w^{(2)}=\frac{-\mu_{1} b_{z}}{\pi\left(\mu_{1}+\mu_{2}\right)}\left[\Psi^{-}\left((r / d)^{n}, n(\theta-\alpha)\right)-\Psi^{-}\left((r / d)^{n}, n(\theta+\alpha)\right)\right] \\
& \tau_{r z}^{(2)}=\frac{n \mu_{1} \mu_{2} b_{z}}{\pi\left(\mu_{1}+\mu_{2}\right) r}\left[\Theta^{-}\left((r / d)^{n}, n(\theta-\alpha)\right)-\Theta^{-}\left((r / d)^{n}, n(\theta+\alpha)\right)\right]
\end{aligned}
$$




$$
\tau_{\theta z}^{(2)}=\frac{-n \mu_{1} \mu_{2} b_{z}}{\pi\left(\mu_{1}+\mu_{2}\right) r}\left[\Gamma^{-}\left((r / d)^{n}, n(\theta-\alpha)\right)-\Gamma^{-}\left((r / d)^{n}, n(\theta+\alpha)\right)\right]
$$

where

$$
\Psi^{ \pm}(R, \Phi)=\tan ^{-1} \frac{(R \mp 1) \sin \Phi}{(R \pm 1)(1+\cos \Phi)}
$$

It is surprising to note that the exact full-field solutions for materials 1 and 2 consist of only four and two terms, respectively. Each term is only a combination of simple trigonometric functions. These basic solutions will be used to construct the analytical solutions for the composite wedge with finite radius and the finite annular composite wedge problems in the next two sections. The asymptotic displacement and stress fields near the wedge apex for applying a concentrated load can be easily derived from Eqs. (14) and (15) by taking the limit $r \rightarrow 0$. The singular fields near the wedge apex of material 1 are

$$
\begin{aligned}
& \lim _{r \rightarrow 0} w^{(1)}(r, \theta)=-\frac{\mu_{2} f_{z}}{\pi \mu_{1}\left(\mu_{1}+\mu_{2}\right)}(r / d)^{n}\{\cos (n(\theta-\alpha))+\cos (n(\theta+\alpha))\} \\
& \lim _{r \rightarrow 0} \tau_{r z}^{(1)}(r, \theta)=-\frac{n \mu_{2} f_{z}}{\pi\left(\mu_{1}+\mu_{2}\right) r}(r / d)^{n}\{\cos (n(\theta-\alpha))+\cos (n(\theta+\alpha))\} \\
& \lim _{r \rightarrow 0} \tau_{\theta z}^{(1)}(r, \theta)=\frac{n \mu_{2} f_{z}}{\pi\left(\mu_{1}+\mu_{2}\right) r}(r / d)^{n}\{\sin (n(\theta-\alpha))+\sin (n(\theta+\alpha))\}
\end{aligned}
$$

For material 2, the singular fields are

$$
\begin{aligned}
& \lim _{r \rightarrow 0} w^{(2)}(r, \theta)=-\frac{f_{z}}{\pi\left(\mu_{1}+\mu_{2}\right)}(r / d)^{n}\{\cos (n(\theta-\alpha))+\cos (n(\theta+\alpha))\} \\
& \lim _{r \rightarrow 0} \tau_{r z}^{(2)}(r, \theta)=-\frac{n \mu_{2} f_{z}}{\pi\left(\mu_{1}+\mu_{2}\right) r}(r / d)^{n}\{\cos (n(\theta-\alpha))+\cos (n(\theta+\alpha))\} \\
& \lim _{r \rightarrow 0} \tau_{\theta z}^{(2)}(r, \theta)=\frac{n \mu_{2} f_{z}}{\pi\left(\mu_{1}+\mu_{2}\right) r}(r / d)^{n}\{\sin (n(\theta-\alpha))+\sin (n(\theta+\alpha))\}
\end{aligned}
$$

It is clearly shown in Eqs. (19) and (20) that the order of the stress singularity is $1-\pi / \beta$ and is independent of the two material constants. The stress fields are bounded for the composite wedge with $0<\beta<\pi$. The angular dependence of displacement and stresses near the wedge apes as presented in Eqs. (19) and (20) are the same as those obtained by Ma and Hour (1989).

For the special case of a semi-infinite interface crack, i.e., $n=1 / 2$, the solutions of shear stress $\tau_{\theta z}$ for materials 1 and 2 for applying a concentrated load are reduced to simple formulations as follows

$$
\begin{aligned}
\tau_{\theta z}^{(1)}= & \frac{f_{z}}{4 \pi r}\left[\frac{(r / d)^{1 / 2} \sin ((\theta-\alpha) / 2)}{1-2(r / d)^{1 / 2} \cos ((\theta-\alpha) / 2)+r / d}+\frac{(r / d)^{1 / 2} \sin ((\theta+\alpha) / 2)}{1-2(r / d)^{1 / 2} \cos ((\theta+\alpha) / 2)+r / d}\right. \\
& \left.-\frac{k(r / d)^{1 / 2} \sin ((\theta-\alpha) / 2)}{1+2(r / d)^{1 / 2} \cos ((\theta-\alpha) / 2)+r / d}-\frac{k(r / d)^{1 / 2} \sin ((\theta+\alpha) / 2)}{1+2(r / d)^{1 / 2} \cos ((\theta+\alpha) / 2)+r / d}\right] \\
\tau_{\theta z}^{(2)}= & \frac{\mu_{2} f_{z}}{2 \pi\left(\mu_{1}+\mu_{2}\right) r}\left[\frac{(r / d)^{1 / 2} \sin ((\theta-\alpha) / 2)}{1-2(r / d)^{1 / 2} \cos ((\theta-\alpha) / 2)+r / d}+\frac{(r / d)^{1 / 2} \sin ((\theta+\alpha) / 2)}{1-2(r / d)^{1 / 2} \cos ((\theta+\alpha) / 2)+r / d}\right]
\end{aligned}
$$


The solutions for applying a screw dislocation are

$$
\begin{aligned}
\tau_{\theta z}^{(1)}= & \frac{\mu_{1} b_{z}}{4 \pi r}\left[-\frac{r / d-(r / d)^{1 / 2} \cos ((\theta-\alpha) / 2)}{1-2(r / d)^{1 / 2} \cos ((\theta-\alpha) / 2)+r / d}+\frac{r / d-(r / d)^{1 / 2} \cos ((\theta+\alpha) / 2)}{1-2(r / d)^{1 / 2} \cos ((\theta+\alpha) / 2)+r / d}\right. \\
& \left.-k \frac{r / d+(r / d)^{1 / 2} \cos ((\theta-\alpha) / 2)}{1+2(r / d)^{1 / 2} \cos ((\theta-\alpha) / 2)+r / d}+k \frac{r / d+(r / d)^{1 / 2} \cos ((\theta+\alpha) / 2)}{1+2(r / d)^{1 / 2} \cos ((\theta+\alpha) / 2)+r / d}\right] \\
\tau_{\theta z}^{(2)}= & \frac{\mu_{1} \mu_{2} b_{z}}{2 \pi\left(\mu_{1}+\mu_{2}\right) r}\left[\frac{r / d-(r / d)^{1 / 2} \cos ((\theta-\alpha) / 2)}{1-2(r / d)^{1 / 2} \cos ((\theta-\alpha) / 2)+r / d}-\frac{r / d-(r / d)^{1 / 2} \cos ((\theta+\alpha) / 2)}{1-2(r / d)^{1 / 2} \cos ((\theta+\alpha) / 2)+r / d}\right]
\end{aligned}
$$

The well-known result of square root singularity near the interface crack tip is clearly indicated in Eqs. (21) and (22). The corresponding result of mode III stress intensity factor can be derived from Eqs. (21) and (22) and are expressed as

$$
\begin{aligned}
& K_{\mathrm{III}}=\left.\lim _{r \rightarrow 0} \sqrt{2 \pi r} \tau_{\theta z}\right|_{\theta=\pi}=\frac{f_{z}}{\sqrt{2 \pi d}}(1-k) \cos (\alpha / 2) \\
& K_{\mathrm{III}}=\left.\lim _{r \rightarrow 0} \sqrt{2 \pi r} \tau_{\theta z}\right|_{\theta=\pi}=\frac{\mu_{1} b_{z}}{\sqrt{2 \pi d}}(1-k) \sin (\alpha / 2)
\end{aligned}
$$

It is noted that for special apex angles, i.e. $\beta=\pi / n$, where $n$ is an integer, the solutions expressed in Eqs. (14) and (15) can be decomposed into a finite number of Green's functions of an infinite plane subjected to concentrated forces. For these special angles, the Green's function of the infinite composite wedge problem can be obtained by the method of image. The numbers $(N)$ and locations $(r, \theta)$ of image singularities of materials 1 and 2 can be expressed as follows:

For material 1,

$$
\left\{\begin{array}{l}
N=4 n-1 \\
(r, \theta)=\left\langle\begin{array}{l}
(d,-\alpha) \\
(d, m \beta \pm \alpha)
\end{array} \quad m=1,2, \ldots, 2 n-1\right.
\end{array}\right.
$$

and for material 2,

$$
\left\{\begin{array}{l}
N=2 n \\
(r, \theta)=(d, 2 m \beta \pm \alpha) \quad m=0,1, \ldots, n-1
\end{array}\right.
$$

In fact, the numbers and locations of image singularities of material 1 and 2 are dependent only on the apex angle of the composite wedge. For example, the geometry configuration of image singularities for material 1 and material 2 of the composite wedge with apex angle $\beta=90^{\circ}$ are shown in Figs. 3 and 4, respectively. There are seven and four image singularities for material 1 and material 2, respectively. Therefore, the fullfiled solutions of a composite sharp wedge with special apex angles can also be represented by a series with finite terms. For material 1, the solutions of series form can be summarized as

$$
\begin{aligned}
w^{(1)}= & \frac{f_{z}}{4 \pi \mu_{1}} \sum_{m=1}^{n}\left[\Omega^{-}(r / d, \theta-2(m-1) \pi / n+\alpha)+\Omega^{-}(r / d, \theta-2(m-1) \pi / n-\alpha)\right. \\
& \left.+k \Omega^{-}(r / d, \theta-(2 m-1) \pi / n+\alpha)+k \Omega^{-}(r / d, \theta-(2 m-1) \pi / n-\alpha)\right]
\end{aligned}
$$




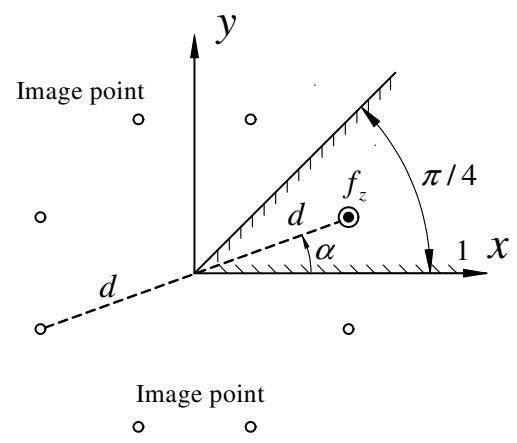

Fig. 3. The locations of image singularities for wedge 1 with an apex angle $\beta / 2=45^{\circ}$.

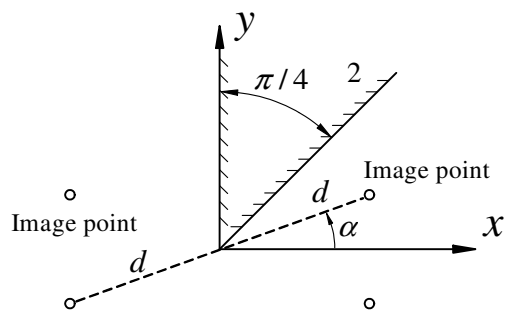

Fig. 4. The locations of image singularities for wedge 2 with an apex angle $\beta / 2=45^{\circ}$.

$$
\begin{aligned}
\tau_{r z}^{(1)}= & \frac{f_{z}}{2 \pi r} \sum_{m=1}^{n}\left[\Gamma^{-}(r / d, \theta-2(m-1) \pi / n+\alpha)+\Gamma^{-}(r / d, \theta-2(m-1) \pi / n-\alpha)\right. \\
& \left.+k \Gamma^{-}(r / d, \theta-(2 m-1) \pi / n+\alpha)+k \Gamma^{-}(r / d, \theta-(2 m-1) \pi / n-\alpha)\right] \\
\tau_{\theta z}^{(1)}= & \frac{f_{z}}{2 \pi r} \sum_{m=1}^{n}\left[\Theta^{-}(r / d, \theta-2(m-1) \pi / n+\alpha)+\Theta^{-}(r / d, \theta-2(m-1) \pi / n-\alpha)\right. \\
& \left.+k \Theta^{-}(r / d, \theta-(2 m-1) \pi / n+\alpha)+k \Theta^{-}(r / d, \theta-(2 m-1) \pi / n-\alpha)\right]
\end{aligned}
$$

For material 2, the solutions are

$$
\begin{aligned}
w^{(2)} & =\frac{f_{z}}{2 \pi\left(\mu_{1}+\mu_{2}\right)} \sum_{m=1}^{n}\left[\Omega^{-}(r / d, \theta-2(m-1) \pi / n+\alpha)+\Omega^{-}(r / d, \theta-2(m-1) \pi / n-\alpha)\right] \\
\tau_{r z}^{(2)} & =\frac{\mu_{2} f_{z}}{\pi\left(\mu_{1}+\mu_{2}\right) r} \sum_{m=1}^{n}\left[\Gamma^{-}(r / d, \theta-2(m-1) \pi / n+\alpha)+\Gamma^{-}(r / d, \theta-2(m-1) \pi / n-\alpha)\right] \\
\tau_{\theta z}^{(2)} & =\frac{\mu_{2} f_{z}}{\pi\left(\mu_{1}+\mu_{2}\right) r} \sum_{m=1}^{n}\left[\Theta^{-}(r / d, \theta-2(m-1) \pi / n+\alpha)+\Theta^{-}(r / d, \theta-2(m-1) \pi / n-\alpha)\right]
\end{aligned}
$$

Eqs. (27) and (28) present the solutions for the same problem as that in Eqs. (14) and (15) for the special case that $n$ is an integer. Obviously, the solutions in Eqs. (14) and (15) have simple forms and are also valid for the case that $n$ is not an integer. 


\subsection{Fixed-fixed boundary condition}

By using the similar method, the solutions of composite wedge with fixed displacement boundary condition at the two edges, i.e., $\theta=0$ and $\theta=\beta$, subjected to a concentrated load can be obtained and summarized as follows

$$
\begin{aligned}
& w^{(1)}=\frac{f_{z}}{4 \pi \mu_{1}}\left[\Omega^{-}\left((r / d)^{n}, n(\theta-\alpha)\right)-\Omega^{-}\left((r / d)^{n}, n(\theta+\alpha)\right)-k \Omega^{+}\left((r / d)^{n}, n(\theta-\alpha)\right)+k \Omega^{+}\left((r / d)^{n}, n(\theta+\alpha)\right)\right] \\
& \tau_{r z}^{(1)}=\frac{n f_{z}}{2 \pi r}\left[\Gamma^{-}\left((r / d)^{n}, n(\theta-\alpha)\right)-\Gamma^{-}\left((r / d)^{n}, n(\theta+\alpha)\right)-k \Gamma^{+}\left((r / d)^{n}, n(\theta-\alpha)\right)+k \Gamma^{+}\left((r / d)^{n}, n(\theta+\alpha)\right)\right] \\
& \tau_{\theta z}^{(1)}=\frac{n f_{z}}{2 \pi r}\left[\Theta^{-}\left((r / d)^{n}, n(\theta-\alpha)\right)-\Theta^{-}\left((r / d)^{n}, n(\theta+\alpha)\right)+k \Theta^{+}\left((r / d)^{n}, n(\theta-\alpha)\right)-k \Theta^{+}\left((r / d)^{n}, n(\theta+\alpha)\right)\right]
\end{aligned}
$$

for material 1, and

$$
\begin{aligned}
w^{(2)} & =\frac{f_{z}}{2 \pi\left(\mu_{1}+\mu_{2}\right)}\left[\Omega^{-}\left((r / d)^{n}, n(\theta-\alpha)\right)-\Omega^{-}\left((r / d)^{n}, n(\theta+\alpha)\right)\right] \\
\tau_{r z}^{(2)} & =\frac{n \mu_{2} f_{z}}{\pi\left(\mu_{1}+\mu_{2}\right) r}\left[\Gamma^{-}\left((r / d)^{n}, n(\theta-\alpha)\right)-\Gamma^{-}\left((r / d)^{n}, n(\theta+\alpha)\right)\right] \\
\tau_{\theta z}^{(2)} & =\frac{n \mu_{2} f_{z}}{\pi\left(\mu_{1}+\mu_{2}\right) r}\left[\Theta^{-}\left((r / d)^{n}, n(\theta-\alpha)\right)-\Theta^{-}\left((r / d)^{n}, n(\theta+\alpha)\right)\right]
\end{aligned}
$$

for material 2. The solutions for applying a screw dislocation are

$$
\begin{aligned}
& w^{(1)}=\frac{-b_{z}}{2 \pi}\left[\Psi^{-}\left((r / d)^{n}, n(\theta-\alpha)\right)+\Psi^{-}\left((r / d)^{n}, n(\theta+\alpha)\right)-k \Psi^{+}\left((r / d)^{n}, n(\theta-\alpha)\right)-k \Psi^{+}\left((r / d)^{n}, n(\theta+\alpha)\right)\right] \\
& \tau_{r z}^{(1)}=\frac{n \mu_{1} b_{z}}{2 \pi r}\left[\Theta^{-}\left((r / d)^{n}, n(\theta-\alpha)\right)+\Theta^{-}\left((r / d)^{n}, n(\theta+\alpha)\right)+k \Theta^{+}\left((r / d)^{n}, n(\theta-\alpha)\right)+k \Theta^{+}\left((r / d)^{n}, n(\theta+\alpha)\right)\right] \\
& \tau_{\theta z}^{(1)}=\frac{-n \mu_{1} b_{z}}{2 \pi r}\left[\Gamma^{-}\left((r / d)^{n}, n(\theta-\alpha)\right)+\Gamma^{-}\left((r / d)^{n}, n(\theta+\alpha)\right)-k \Gamma^{+}\left((r / d)^{n}, n(\theta-\alpha)\right)-k \Gamma^{+}\left((r / d)^{n}, n(\theta+\alpha)\right)\right]
\end{aligned}
$$

for material 1, and

$$
\begin{aligned}
& w^{(2)}=\frac{-\mu_{1} b_{z}}{\pi\left(\mu_{1}+\mu_{2}\right)}\left[\Psi^{-}\left((r / d)^{n}, n(\theta-\alpha)\right)+\Psi^{-}\left((r / d)^{n}, n(\theta+\alpha)\right)\right] \\
& \tau_{r z}^{(2)}=\frac{n \mu_{1} \mu_{2} b_{z}}{\pi\left(\mu_{1}+\mu_{2}\right) r}\left[\Theta^{-}\left((r / d)^{n}, n(\theta-\alpha)\right)+\Theta^{-}\left((r / d)^{n}, n(\theta+\alpha)\right)\right]
\end{aligned}
$$




$$
\tau_{\theta z}^{(2)}=\frac{-n \mu_{1} \mu_{2} b_{z}}{\pi\left(\mu_{1}+\mu_{2}\right) r}\left[\Gamma^{-}\left((r / d)^{n}, n(\theta-\alpha)\right)+\Gamma^{-}\left((r / d)^{n}, n(\theta+\alpha)\right)\right]
$$

for material 2. It is interesting to note that the elementary functions appear in the solutions are the same for the free-free (Eqs. (14)-(17)) and fixed-fixed (Eqs. (27)-(30)) boundary conditions.

\section{Composite wedge with a finite radius and the method of image}

Consider an isotropic composite wedge with a finite radius $b$ and equal apex angle $\beta / 2$ subjected to a concentrated force $f_{z}$ applied at the location $(r, \theta)=(d, \alpha)$ as shown in Fig. 5. In order to obtain the closedform solution of this problem without using the mathematics derivation, the image method for circular configuration is used. Based on the result presented by Lin and Ma (2003) for the problem of single wedge with finite radius that for the loading applied at the location $(d, \alpha)$, the location of the corresponding image singularity for the circular boundary is $\left(b^{2} / d, \alpha\right)$ and is indicated in Fig. 6 . The magnitude of the image singularity depends on the boundary condition at the circumference segment. The summation of the solutions for the applied loading at $(d, \alpha)$ and $\left(b^{2} / d, \alpha\right)$ (the image point) for the composite wedge with infinite length will satisfy the boundary condition (traction free or fixed) on the circular segment $r=b$. Since the analytical solution for composite wedge with infinite length has been developed in the previous section, the solution for the composite wedge with a finite radius can be easily obtained without difficulty. The solutions of the stress field $\tau_{\theta z}$ are presented for various boundary condition as follows.

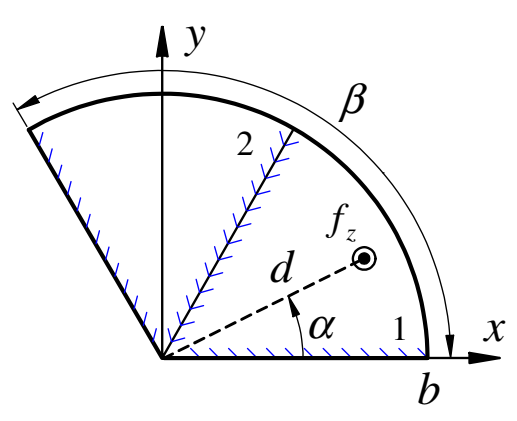

Fig. 5. Schematic representation of a concentrated force applied in a composite sharp wedge with a finite radius.

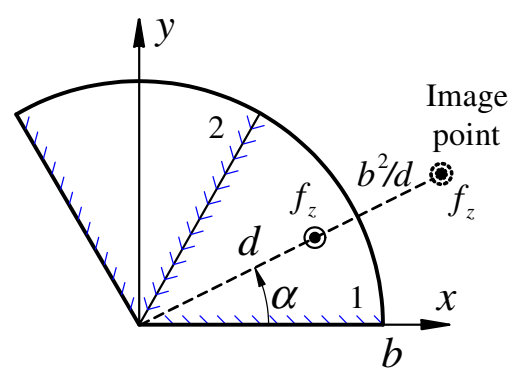

Fig. 6. The location of the image singularity with respect to the circular boundary. 


\subsection{Free-free-free boundary condition}

Consider the wedge are traction free for all boundaries, and one pair of self-equilibrium forces $f_{z}$ are applied on the wedge at locations $(r, \theta)=\left(d_{1}, \alpha_{1}\right)$ and $\left(d_{2}, \alpha_{2}\right)$ in material 1 . The solutions for shear stress $\tau_{\theta z}$ are

$$
\begin{aligned}
\tau_{\theta z}^{(1)}= & \frac{n f_{z}}{2 \pi r} \sum_{m=1}^{2}(-1)^{m+1}\left[\Theta^{-}\left(\left(r / d_{m}\right)^{n}, n\left(\theta-\alpha_{m}\right)\right)+\Theta^{-}\left(\left(r / d_{m}\right)^{n}, n\left(\theta+\alpha_{m}\right)\right)-k \Theta^{+}\left(\left(r / d_{m}\right)^{n}, n\left(\theta-\alpha_{m}\right)\right)\right. \\
& -k \Theta^{+}\left(\left(r / d_{m}\right)^{n}, n\left(\theta+\alpha_{m}\right)\right)+\Theta^{-}\left(\left(r d_{m} / b^{2}\right)^{n}, n\left(\theta-\alpha_{m}\right)\right)+\Theta^{-}\left(\left(r d_{m} / b^{2}\right)^{n}, n\left(\theta+\alpha_{m}\right)\right) \\
& \left.-k \Theta^{+}\left(\left(r d_{m} / b^{2}\right)^{n}, n\left(\theta-\alpha_{m}\right)\right)-k \Theta^{+}\left(\left(r d_{m} / b^{2}\right)^{n}, n\left(\theta+\alpha_{m}\right)\right)\right]
\end{aligned}
$$

for material 1 , and

$$
\begin{aligned}
\tau_{\theta z}^{(2)}= & \frac{n \mu_{2} f_{z}}{\pi\left(\mu_{1}+\mu_{2}\right) r} \sum_{m=1}^{2}(-1)^{m+1}\left[\Theta^{-}\left(\left(r / d_{m}\right)^{n}, n\left(\theta-\alpha_{m}\right)\right)+\Theta^{-}\left(\left(r / d_{m}\right)^{n}, n\left(\theta+\alpha_{m}\right)\right)\right. \\
& \left.+\Theta^{-}\left(\left(r d_{m} / b^{2}\right)^{n}, n\left(\theta-\alpha_{m}\right)\right)+\Theta^{-}\left(\left(r d_{m} / b^{2}\right)^{n}, n\left(\theta+\alpha_{m}\right)\right)\right]
\end{aligned}
$$

for material 2. For a special apex angle that $n=1 / 2$, which represents the problem of a composite circular disk with an interface crack length $b$, the associated shear stresses $\tau_{\theta z}$ are

$$
\begin{aligned}
\tau_{\theta z}^{(1)}= & \frac{f_{z}}{4 \pi r} \sum_{m=1}^{2}(-1)^{m+1}\left[\Theta^{-}\left(\left(r / d_{m}\right)^{1 / 2},\left(\theta-\alpha_{m}\right) / 2\right)+\Theta^{-}\left(\left(r / d_{m}\right)^{1 / 2},\left(\theta+\alpha_{m}\right) / 2\right)-k \Theta^{+}\left(\left(r / d_{m}\right)^{1 / 2},\right.\right. \\
& \left.\left(\theta-\alpha_{m}\right) / 2\right)-k \Theta^{+}\left(\left(r / d_{m}\right)^{1 / 2},\left(\theta+\alpha_{m}\right) / 2\right)+\Theta^{-}\left(\left(r d_{m} / b^{2}\right)^{1 / 2},\left(\theta-\alpha_{m}\right) / 2\right)+\Theta^{-}\left(\left(r d_{m} / b^{2}\right)^{1 / 2},\right. \\
& \left.\left.\left(\theta+\alpha_{m}\right) / 2\right)-k \Theta^{+}\left(\left(r d_{m} / b^{2}\right)^{1 / 2},\left(\theta-\alpha_{m}\right) / 2\right)-k \Theta^{+}\left(\left(r d_{m} / b^{2}\right)^{1 / 2},\left(\theta+\alpha_{m}\right) / 2\right)\right] \\
\tau_{\theta z}^{(2)}= & \frac{\mu_{2} f_{z}}{2 \pi\left(\mu_{1}+\mu_{2}\right) r} \sum_{m=1}^{2}(-1)^{m+1}\left[\Theta^{-}\left(\left(r / d_{m}\right)^{1 / 2},\left(\theta-\alpha_{m}\right) / 2\right)+\Theta^{-}\left(\left(r / d_{m}\right)^{1 / 2},\left(\theta+\alpha_{m}\right) / 2\right)\right. \\
& \left.+\Theta^{-}\left(\left(r d_{m} / b^{2}\right)^{1 / 2},\left(\theta-\alpha_{m}\right) / 2\right)+\Theta^{-}\left(\left(r d_{m} / b^{2}\right)^{1 / 2},\left(\theta+\alpha_{m}\right) / 2\right)\right]
\end{aligned}
$$

The corresponding stress intensity factor of this problem is

$$
K_{\mathrm{III}}=\left.\lim _{r \rightarrow 0} \sqrt{2 \pi r} \tau_{\theta z}\right|_{\theta=\pi}=\frac{f_{z}}{\sqrt{2 \pi d}}(1-k)\left[\left(1+d_{1} / b\right) \cos \left(\alpha_{1} / 2\right)-\left(1+d_{2} / b\right) \cos \left(\alpha_{2} / 2\right)\right]
$$

For the problem that a screw dislocation with Burger's vector $b_{z}$ is applied at the location $(r, \theta)=(d, \alpha)$ in material 1. The solutions for shear stress $\tau_{\theta z}$ are

$$
\begin{aligned}
\tau_{\theta z}^{(1)}= & \frac{-n \mu_{1} b_{z}}{2 \pi r}\left[\Gamma^{-}\left((r / d)^{n}, n(\theta-\alpha)\right)-\Gamma^{-}\left((r / d)^{n}, n(\theta+\alpha)\right)+k \Gamma^{+}\left((r / d)^{n}, n(\theta-\alpha)\right)\right. \\
& -k \Gamma^{+}\left((r / d)^{n}, n(\theta+\alpha)\right)-\Gamma^{-}\left(\left(r d / b^{2}\right)^{n}, n(\theta-\alpha)\right)+\Gamma^{-}\left(\left(r d / b^{2}\right)^{n}, n(\theta+\alpha)\right) \\
& \left.-k \Gamma^{+}\left(\left(r d / b^{2}\right)^{n}, n(\theta-\alpha)\right)+k \Gamma^{+}\left(\left(r d / b^{2}\right)^{n}, n(\theta+\alpha)\right)\right]
\end{aligned}
$$

for material 1 , and

$$
\begin{aligned}
\tau_{\theta z}^{(2)}= & \frac{-n \mu_{1} \mu_{2} b_{z}}{\pi\left(\mu_{1}+\mu_{2}\right) r}\left[\Gamma^{-}\left((r / d)^{n}, n(\theta-\alpha)\right)-\Gamma^{-}\left((r / d)^{n}, n(\theta+\alpha)\right)-\Gamma^{-}\left(\left(r d / b^{2}\right)^{n}, n(\theta-\alpha)\right)\right. \\
& \left.+\Gamma^{-}\left(\left(r d / b^{2}\right)^{n}, n(\theta+\alpha)\right)\right]
\end{aligned}
$$


for material 2. For a special apex angle that $\beta=2 \pi(n=1 / 2)$, the associated shear stresses $\tau_{\theta z}$ are

$$
\begin{aligned}
\tau_{\theta z}^{(1)}= & \frac{-\mu_{1} b_{z}}{4 \pi r}\left[\Gamma^{-}\left((r / d)^{1 / 2},(\theta-\alpha) / 2\right)-\Gamma^{-}\left((r / d)^{1 / 2},(\theta+\alpha) / 2\right)+k \Gamma^{+}\left((r / d)^{1 / 2},(\theta-\alpha) / 2\right)\right. \\
& -k \Gamma^{+}\left((r / d)^{1 / 2},\left(\theta+\alpha_{m}\right) / 2\right)-\Gamma^{-}\left(\left(r d / b^{2}\right)^{1 / 2},(\theta-\alpha) / 2\right)+\Gamma^{-}\left(\left(r d / b^{2}\right)^{1 / 2},(\theta+\alpha) / 2\right) \\
& \left.-k \Gamma^{+}\left(\left(r d / b^{2}\right)^{1 / 2},(\theta-\alpha) / 2\right)+k \Gamma^{+}\left(\left(r d / b^{2}\right)^{1 / 2},(\theta+\alpha) / 2\right)\right] \\
\tau_{\theta z}^{(2)}= & \frac{-\mu_{1} \mu_{2} b_{z}}{2 \pi\left(\mu_{1}+\mu_{2}\right) r}\left[\Gamma^{-}\left((r / d)^{1 / 2},(\theta-\alpha) / 2\right)-\Gamma^{-}\left((r / d)^{1 / 2},(\theta+\alpha) / 2\right)-\Gamma^{-}\left(\left(r d / b^{2}\right)^{1 / 2},(\theta-\alpha) / 2\right)\right. \\
& \left.+\Gamma^{-}\left(\left(r d / b^{2}\right)^{1 / 2},(\theta+\alpha) / 2\right)\right]
\end{aligned}
$$

The corresponding stress intensity factor for this problem is

$$
K_{\mathrm{III}}=\left.\lim _{r \rightarrow 0} \sqrt{2 \pi r} \tau_{\theta z}\right|_{\theta=\pi}=\frac{\mu_{1} b_{z}}{\sqrt{2 \pi d}}(1-k)(1-d / b) \sin (\alpha / 2)
$$

\subsection{Free-free-fixed boundary condition}

Consider the composite wedge is fixed along the circular segment $r=b$ and is traction free on the radial edges $\theta=0$ and $\theta=\beta$. A concentrated loading $f_{z}$ is applied at the location $(r, \theta)=(d, \alpha)$. The full-filed solution for material 1 is

$$
\begin{aligned}
\tau_{\theta z}^{(1)}= & \frac{n f_{z}}{2 \pi r}\left[\Theta^{-}\left((r / d)^{n}, n(\theta-\alpha)\right)+\Theta^{-}\left((r / d)^{n}, n(\theta+\alpha)\right)-k \Theta^{+}\left((r / d)^{n}, n(\theta-\alpha)\right)\right. \\
& -k \Theta^{+}\left((r / d)^{n}, n(\theta+\alpha)\right)-\Theta^{-}\left(\left(r d / b^{2}\right)^{n}, n(\theta-\alpha)\right)-\Theta^{-}\left(\left(r d / b^{2}\right)^{n}, n(\theta+\alpha)\right) \\
& \left.+k \Theta^{+}\left(\left(r d / b^{2}\right)^{n}, n(\theta-\alpha)\right)+k \Theta^{+}\left(\left(r d / b^{2}\right)^{n}, n(\theta+\alpha)\right)\right]
\end{aligned}
$$

For material 2, the solution is

$$
\begin{aligned}
\tau_{\theta z}^{(2)}= & \frac{n \mu_{2} f_{z}}{\pi\left(\mu_{1}+\mu_{2}\right) r}\left[\Theta^{-}\left((r / d)^{n}, n(\theta-\alpha)\right)+\Theta^{-}\left((r / d)^{n}, n(\theta+\alpha)\right)-\Theta^{-}\left(\left(r d / b^{2}\right)^{n}, n(\theta-\alpha)\right)\right. \\
& \left.-\Theta^{-}\left(\left(r d / b^{2}\right)^{n}, n(\theta+\alpha)\right)\right]
\end{aligned}
$$

For the special case that $n=1 / 2$, a composite circular disk with an interface crack length $b$, the stress intensity factor is

$$
K_{\mathrm{III}}=\left.\lim _{r \rightarrow 0} \sqrt{2 \pi r} \tau_{\theta z}\right|_{\theta=\pi}=\frac{f_{z}}{\sqrt{2 \pi d}}(1-k)(1-d / b) \cos (\alpha / 2)
$$

\subsection{Fixed-fixed-free boundary condition}

Let the composite wedge be traction free at the boundary $r=b$, and the boundaries $\theta=0$ and $\theta=\beta$ are fixed. The concentrated loading $f_{z}$ (or the screw dislocation $b_{z}$ ) is applied at the location $(r, \theta)=(d, \alpha)$, the full-field solutions are 


$$
\begin{aligned}
\tau_{\theta z}^{(1)}= & \frac{n f_{z}}{2 \pi r}\left[\Theta^{-}\left((r / d)^{n}, n(\theta-\alpha)\right)-\Theta^{-}\left((r / d)^{n}, n(\theta+\alpha)\right)+k \Theta^{+}\left((r / d)^{n}, n(\theta-\alpha)\right)\right. \\
& -k \Theta^{+}\left((r / d)^{n}, n(\theta+\alpha)\right)+\Theta^{-}\left(\left(r d / b^{2}\right)^{n}, n(\theta-\alpha)\right)-\Theta^{-}\left(\left(r d / b^{2}\right)^{n}, n(\theta+\alpha)\right) \\
& \left.+k \Theta^{+}\left(\left(r d / b^{2}\right)^{n}, n(\theta-\alpha)\right)-k \Theta^{+}\left(\left(r d / b^{2}\right)^{n}, n(\theta+\alpha)\right)\right] \\
\tau_{\theta z}^{(1)}= & \frac{-n \mu_{1} b_{z}}{2 \pi r}\left[\Gamma^{-}\left((r / d)^{n}, n(\theta-\alpha)\right)+\Gamma^{-}\left((r / d)^{n}, n(\theta+\alpha)\right)-k \Gamma^{+}\left((r / d)^{n}, n(\theta-\alpha)\right)\right. \\
& -k \Gamma^{+}\left((r / d)^{n}, n(\theta+\alpha)\right)-\Gamma^{-}\left(\left(r d / b^{2}\right)^{n}, n(\theta-\alpha)\right)-\Gamma^{-}\left(\left(r d / b^{2}\right)^{n}, n(\theta+\alpha)\right) \\
& \left.+k \Gamma^{+}\left(\left(r d / b^{2}\right)^{n}, n(\theta-\alpha)\right)+k \Gamma^{+}\left(\left(r d / b^{2}\right)^{n}, n(\theta+\alpha)\right)\right]
\end{aligned}
$$

for material 1 , and

$$
\begin{aligned}
\tau_{\theta z}^{(2)}= & \frac{n \mu_{2} f_{z}}{\pi\left(\mu_{1}+\mu_{2}\right) r}\left[\Theta^{-}\left((r / d)^{n}, n(\theta-\alpha)\right)-\Theta^{-}\left((r / d)^{n}, n(\theta+\alpha)\right)+\Theta^{-}\left(\left(r d / b^{2}\right)^{n}, n(\theta-\alpha)\right)\right. \\
& \left.-\Theta^{-}\left(\left(r d / b^{2}\right)^{n}, n(\theta+\alpha)\right)\right] \\
\tau_{\theta z}^{(2)}= & \frac{-n \mu_{1} \mu_{2} b_{z}}{\pi\left(\mu_{1}+\mu_{2}\right) r}\left[\Gamma^{-}\left((r / d)^{n}, n(\theta-\alpha)\right)+\Gamma^{-}\left((r / d)^{n}, n(\theta+\alpha)\right)-\Gamma^{-}\left(\left(r d / b^{2}\right)^{n}, n(\theta-\alpha)\right)\right. \\
& \left.-\Gamma^{-}\left(\left(r d / b^{2}\right)^{n}, n(\theta+\alpha)\right)\right]
\end{aligned}
$$

for material 2. For the special case that $n=1 / 2$, a composite circular disk with an interface crack length $b$, the stress intensity factor is

$$
\begin{aligned}
& K_{\mathrm{III}}=\left.\lim _{r \rightarrow 0} \sqrt{2 \pi r} \tau_{r z}\right|_{\theta=\pi}=\frac{f_{z}}{\sqrt{2 \pi d}}(1-k)(1+d / b) \sin (\alpha / 2) \\
& K_{\mathrm{III}}=\left.\lim _{r \rightarrow 0} \sqrt{2 \pi r} \tau_{r z}\right|_{\theta=\pi}=\frac{\mu_{1} b_{z}}{\sqrt{2 \pi d}}(1+k)(1-d / b) \cos (\alpha / 2)
\end{aligned}
$$

\subsection{Fixed-fixed-fixed boundary condition}

The composite wedge is fixed for all boundaries, a concentrated forces $f_{z}$ is applied on the composite wedge at the location $(r, \theta)=(d, \alpha)$. The solution of material 1 is

$$
\begin{aligned}
\tau_{\theta z}^{(1)}= & \frac{n f_{z}}{2 \pi r}\left[\Theta^{-}\left((r / d)^{n}, n(\theta-\alpha)\right)-\Theta^{-}\left((r / d)^{n}, n(\theta+\alpha)\right)+k \Theta^{+}\left((r / d)^{n}, n(\theta-\alpha)\right)\right. \\
& -k \Theta^{+}\left((r / d)^{n}, n(\theta+\alpha)\right)-\Theta^{-}\left(\left(r d / b^{2}\right)^{n}, n(\theta-\alpha)\right)+\Theta^{-}\left(\left(r d / b^{2}\right)^{n}, n(\theta+\alpha)\right) \\
& \left.-k \Theta^{+}\left(\left(r d / b^{2}\right)^{n}, n(\theta-\alpha)\right)+k \Theta^{+}\left(\left(r d / b^{2}\right)^{n}, n(\theta+\alpha)\right)\right]
\end{aligned}
$$

For material 2, it is

$$
\begin{aligned}
\tau_{\theta z}^{(2)}= & \frac{n \mu_{2} f_{z}}{\pi\left(\mu_{1}+\mu_{2}\right) r}\left[\Theta^{-}\left((r / d)^{n}, n(\theta-\alpha)\right)-\Theta^{-}\left((r / d)^{n}, n(\theta+\alpha)\right)-\Theta^{-}\left(\left(r d / b^{2}\right)^{n}, n(\theta-\alpha)\right)\right. \\
& \left.+\Theta^{-}\left(\left(r d / b^{2}\right)^{n}, n(\theta+\alpha)\right)\right]
\end{aligned}
$$

For the special case that $n=1 / 2$, a composite circular disk with an interface crack length $b$, the stress intensity factor is 


$$
K_{\mathrm{III}}=\left.\lim _{r \rightarrow 0} \sqrt{2 \pi r} \tau_{r z}\right|_{\theta=\pi}=\frac{f_{z}}{\sqrt{2 \pi d}}(1-k) \sin (\alpha / 2)(1-d / b)
$$

The full-field solutions of displacement $w$ and shear stress $\tau_{r z}$ are presented in Appendices A and B for applying a concentrated load and a screw dislocation, respectively. The anti-plane deformation of a single isotropic wedge with a finite radius was studied by Kargarnovin et al. (1997) using the finite Mellin transform and the full-field solution was presented by complicated formulation with an infinite series form. However, the explicit full-field solutions for a composite wedge with a finite radius presented in this section only consist of finite terms. The solutions for a single wedge with a finite radius can be easily obtained by setting $\mu_{1}=\mu_{2}=\mu$ and $k=0$. The image method used in this section for the circular boundary will be extended in the next section to solve the more complicated finite annular composite wedge problem.

\section{The annular composite wedge}

Consider an annular composite wedge with equal apex angle $\beta / 2$ and finite radii at $r=a$ and $r=b$ as shown in Fig. 7. The methodology for constructing the analytical solution for this complicated problem is similar to that used in the previous section. By using the closed-form solution of a composite wedge of infinite extent presented in Section 3 and the method of image to satisfy two circular boundaries at $r=a$ and $r=b$, the complete solutions for the composite annular wedge can be easily constructed for various boundary conditions. Since two circular segments are involved in this problem, the infinite number of image singularities should be used to satisfy the two boundary conditions at $r=a$ and $r=b$.

\subsection{Free-free-free-free boundary condition}

The first case considered in this section is an annular composite wedge subjected to one pair of selfequilibrium forces $f_{z}$ at $(r, \theta)=\left(d_{1}, \alpha_{1}\right)$ and $\left(d_{2}, \alpha_{2}\right)$ in material 1 with traction free boundaries. The four boundary conditions are

$$
\begin{cases}\tau_{\theta z}(r, 0)=\tau_{\theta z}(r, \beta)=0 & \text { for } a \leqslant r \leqslant b \\ \tau_{r z}(a, \theta)=\tau_{r z}(b, \theta)=0 & \text { for } 0 \leqslant \theta \leqslant \beta\end{cases}
$$

To avoid the tedious expression of the full-field solutions for this problem, only the stress component $\tau_{\theta z}$ is presented in this section. The full-field solutions of displacement $w$ and stress component $\tau_{r z}$ are summarized in Appendix C. The full-field solutions $\tau_{\theta z}$ are

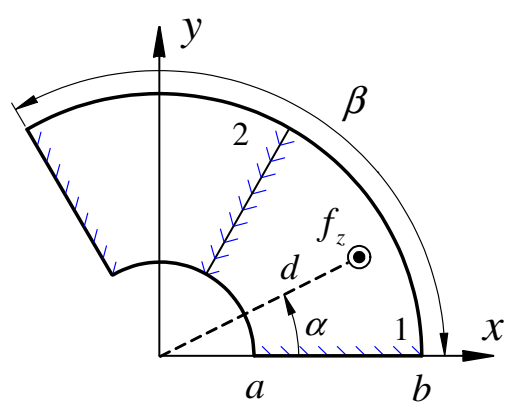

Fig. 7. Schematic diagram of a concentrated force applied in a composite annular wedge. 


$$
\tau_{\theta z}^{(1)}=\frac{n f_{z}}{2 \pi r} \sum_{\ell=0}^{\infty} \sum_{j=1}^{4} \sum_{m=1}^{2}(-1)^{m+1}\left[\Theta^{-}\left(r_{j}, \phi^{-}\right)+\Theta^{-}\left(r_{j}, \phi^{+}\right)-k \Theta^{+}\left(r_{j}, \phi^{-}\right)-k \Theta^{+}\left(r_{j}, \phi^{+}\right)\right]
$$

for material 1 , and

$$
\tau_{\theta z}^{(2)}=\frac{n \mu_{2} f_{z}}{\pi\left(\mu_{1}+\mu_{2}\right) r} \sum_{\ell=0}^{\infty} \sum_{j=1}^{4} \sum_{m=1}^{2}(-1)^{m+1}\left[\Theta^{-}\left(r_{j}, \phi^{-}\right)+\Theta^{-}\left(r_{j}, \phi^{+}\right)\right]
$$

for material 2, where

$$
\left\{\begin{array}{lll}
r_{1}=\left((b / a)^{2 \ell} r / d_{m}\right)^{n}, & r_{2}=\left((b / a)^{2(\ell+1)} r d_{m} / b^{2}\right)^{n}, & \phi^{-}=n\left(\theta-\alpha_{m}\right) \\
r_{3}=\left((a / b)^{2 \ell} r d_{m} / b^{2}\right)^{n}, & r_{4}=\left((a / b)^{2(\ell+1)} r / d_{m}\right)^{n}, & \phi^{+}=n\left(\theta+\alpha_{m}\right)
\end{array}\right.
$$

The explicit solutions presented in Eqs. (54) and (55) are infinite series with only one summation. The solutions in Eqs. (54) and (55) for $\ell=0$ and $j=1$ represent the Green's function of a composite wedge with infinite length which is discussed in the Section 3. All the other terms are superimposed to satisfy the circular boundary conditions along $r=a$ and $r=b$. The locations of image singularities along the radial direction can be determined and summarized as follows:

$$
r=\left\{\begin{array}{lll}
d_{m}(a / b)^{2(\ell+1)}, & (a / b)^{2(\ell+1)}\left(b^{2} / d_{m}\right) & \text { for } r<a \\
d_{m}(b / a)^{2(\ell+1)}, & (b / a)^{2 \ell}\left(b^{2} / d_{m}\right) & \text { for } r>b
\end{array} \quad m=1,2 \text { and } \ell=0,1,2, \ldots, \infty\right.
$$

For the case that an annular composite wedge subjected to a screw dislocation with Burger's vector $b_{z}$ at $(r, \theta)=(d, \alpha)$ in material 1 with traction free boundaries. The full-field solution $\tau_{\theta z}$ is

$$
\tau_{\theta z}^{(1)}=\frac{n \mu_{1} b_{z}}{2 \pi r} \sum_{\ell=0}^{\infty} \sum_{j=1}^{4}(-1)^{j}\left[\Gamma^{-}\left(r_{j}, \phi^{-}\right)-\Gamma^{-}\left(r_{j}, \phi^{+}\right)+k \Gamma^{+}\left(r_{j}, \phi^{-}\right)-k \Gamma^{+}\left(r_{j}, \phi^{+}\right)\right]
$$

for material 1 , and

$$
\tau_{\theta z}^{(2)}=\frac{n \mu_{1} \mu_{2} b_{z}}{\pi\left(\mu_{1}+\mu_{2}\right) r} \sum_{\ell=0}^{\infty} \sum_{j=1}^{4}(-1)^{j}\left[\Gamma^{-}\left(r_{j}, \phi^{-}\right)-\Gamma^{-}\left(r_{j}, \phi^{+}\right)\right]
$$

for material 2, where

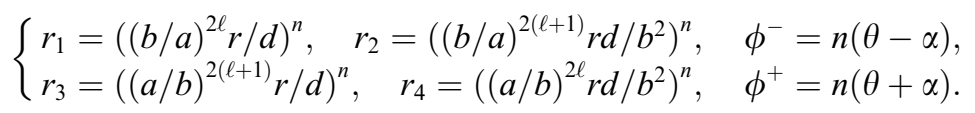

The full-field solutions of displacement $w$ and stress component $\tau_{r z}$ are summarized in Appendix D.

\subsection{Fixed-fixed-free-free boundary condition}

Consider an annular composite wedge with two fixed and two free boundaries subjected to a concentrated force located at $r=d$ and $\theta=\alpha$. For the case that the boundary is fixed along the radial edges and is free along the circular segments, the boundary conditions are

$$
\begin{cases}w(r, 0)=w(r, \beta)=0 & \text { for } a \leqslant r \leqslant b \\ \tau_{r z}(a, \theta)=\tau_{r z}(b, \theta)=0 & \text { for } 0 \leqslant \theta \leqslant \beta\end{cases}
$$

Follow a similar procedure as indicated in the previous case, the full-filed solutions are presented as follows. For material 1, the full-filed solution is 


$$
\tau_{\theta z}^{(1)}=\frac{n f_{z}}{2 \pi r} \sum_{\ell=0}^{\infty} \sum_{j=1}^{4}\left[\Theta^{-}\left(r_{j}, \phi^{-}\right)-\Theta^{-}\left(r_{j}, \phi^{+}\right)+k \Theta^{+}\left(r_{j}, \phi^{-}\right)-k \Theta^{+}\left(r_{j}, \phi^{+}\right)\right]
$$

For material 2,

$$
\tau_{\theta z}^{(2)}=\frac{n \mu_{2} f_{z}}{\pi\left(\mu_{1}+\mu_{2}\right) r} \sum_{\ell=0}^{\infty} \sum_{j=1}^{4}\left[\Theta^{-}\left(r_{j}, \phi^{-}\right)-\Theta^{-}\left(r_{j}, \phi^{+}\right)\right]
$$

where $\Theta^{ \pm}$is the same as that presented in Eq. (15) and

$$
\left\{\begin{array}{lll}
r_{1}=\left((b / a)^{2 \ell} r / d\right)^{n}, & r_{2}=\left((b / a)^{2(\ell+1)} r d / b^{2}\right)^{n}, & \phi^{-}=n(\theta-\alpha) \\
r_{3}=\left((a / b)^{2 \ell} r d / b^{2}\right)^{n}, & r_{4}=\left((a / b)^{2(\ell+1)} r / d\right)^{n}, & \phi^{+}=n(\theta+\alpha)
\end{array}\right.
$$

\subsection{Free-free-fixed-free boundary condition}

An annular composite wedge subjected to a concentrated force is fixed along one radial edge $r=a$ and the other three boundaries are traction free; the boundary conditions are

$$
\begin{cases}\tau_{\theta z}(r, 0)=\tau_{\theta z}(r, \beta)=0 & \text { for } a \leqslant r \leqslant b \\ w(a, \theta)=\tau_{r z}(b, \theta)=0 & \text { for } 0 \leqslant \theta \leqslant \beta\end{cases}
$$

The full-filed solutions of shear stresses are summarized as follows

$$
\begin{aligned}
\tau_{\theta z}^{(1)} & =\frac{n f_{z}}{2 \pi r} \sum_{\ell=0}^{\infty} \sum_{j=1}^{4}(-1)^{\ell+j-1}\left[\Theta^{-}\left(r_{j}, \phi^{-}\right)+\Theta^{-}\left(r_{j}, \phi^{+}\right)-k \Theta^{+}\left(r_{j}, \phi^{-}\right)-k \Theta^{+}\left(r_{j}, \phi^{+}\right)\right] \\
\tau_{\theta z}^{(2)} & =\frac{n \mu_{2} f_{z}}{\pi\left(\mu_{1}+\mu_{2}\right) r} \sum_{\ell=0}^{\infty} \sum_{j=1}^{4}(-1)^{\ell+j-1}\left[\Theta^{-}\left(r_{j}, \phi^{-}\right)+\Theta^{-}\left(r_{j}, \phi^{+}\right)\right]
\end{aligned}
$$

The functions $r_{1}, r_{2}, r_{4}, r_{4}, \phi^{+}$and $\phi^{-}$are defined in Eq. (64).

\subsection{Fixed-fixed-free-fixed boundary condition}

Consider an annular composite wedge with three fixed and one free boundaries subjected to a screw dislocation located at $r=d$ and $\theta=\alpha$ in material 1 . The boundary is free along one radial edge $r=a$ and the other three boundaries are fixed. The boundary conditions are

$$
\begin{cases}w(r, 0)=w(r, \beta)=0 & \text { for } a \leqslant r \leqslant b \\ \tau_{r z}(a, \theta)=w(b, \theta)=0 & \text { for } 0 \leqslant \theta \leqslant \beta\end{cases}
$$

Follow a similar procedure as indicated in the previous case, the full-filed solutions are presented as follows. For material 1, the full-filed solution is

$$
\tau_{\theta z}^{(1)}=\frac{n \mu_{1} b_{z}}{2 \pi r} \sum_{\ell=0}^{\infty} \sum_{j=1}^{4}(-1)^{\ell+j}\left[\Gamma^{-}\left(r_{j}, \phi^{-}\right)+\Gamma^{-}\left(r_{j}, \phi^{+}\right)-k \Gamma^{+}\left(r_{j}, \phi^{-}\right)-k \Gamma^{+}\left(r_{j}, \phi^{+}\right)\right]
$$

For material 2, the solution is 


$$
\tau_{\theta z}^{(2)}=\frac{n \mu_{1} \mu_{2} b_{z}}{\pi\left(\mu_{1}+\mu_{2}\right) r} \sum_{\ell=0}^{\infty} \sum_{j=1}^{4}(-1)^{\ell+j}\left[\Gamma^{-}\left(r_{j}, \phi^{-}\right)+\Gamma^{-}\left(r_{j}, \phi^{+}\right)\right]
$$

\section{Image forces exerted on screw dislocations}

The full-field stress distributions of composite wedges subjected to screw dislocations are analyzed in detail in previous sections. The image forces exerted on screw dislocations will be investigated in this section. According to the Peach-Koehler equation, the image force exerted on the screw dislocation can be obtained from the stress filed at the location of the dislocation minus the self-stresses of the dislocation in an infinite plane. In polar coordinate, the relations between image forces and stress fields are

$$
\left[\begin{array}{l}
F_{r} \\
F_{\theta}
\end{array}\right]=\left[\begin{array}{c}
-\tau_{\theta z}^{i} \\
\tau_{r z}^{i}
\end{array}\right] b_{z}
$$

where $F_{r}$ and $F_{\theta}$ denote the image force exerted on a screw dislocation along the radial and circumferential directions, respectively, and $\tau_{\theta z}^{i}=\tau_{\theta z}-\tau_{\theta z}^{s}, \tau_{r z}^{i}=\tau_{r z}-\tau_{r z}^{s}$ in which $\tau_{\theta z}^{s}$ and $\tau_{r z}^{s}$ are the self-stresses of the screw dislocation. The self-stresses of a screw dislocation in an infinite plane are

$$
\begin{aligned}
\tau_{\theta z}^{s} & =\frac{-\mu b_{z}}{2 \pi} \frac{r-d \cos (\theta-\alpha)}{r^{2}-2 r d \cos (\theta-\alpha)+d^{2}} \\
\tau_{r z}^{s} & =\frac{\mu b_{z}}{2 \pi} \frac{d \sin (\theta-\alpha)}{r^{2}-2 r d \cos (\theta-\alpha)+d^{2}}
\end{aligned}
$$

The image forces exerted on the dislocation for different boundary conditions are summarized as follows.

\subsection{Image forces exerted on screw dislocations for infinite composite wedges}

\subsubsection{Free-free boundary condition}

From Eqs. (16), (71) and (72), the image forces exerted on a screw dislocation located at $(d, \alpha)$ for the traction free boundary condition are

$$
\begin{aligned}
& F_{r}^{(1)}(d, \alpha)=-\frac{\mu_{1} b_{z}^{2}}{4 \pi d} \\
& F_{\theta}^{(1)}(d, \alpha)=-\frac{\mu_{1} b_{z}^{2} n}{4 \pi d}\left(\frac{\cos n \alpha}{\sin n \alpha}-k \frac{\sin n \alpha}{\cos n \alpha}\right)
\end{aligned}
$$

for material 1 , and

$$
\begin{aligned}
& F_{r}^{(2)}(d, \alpha)=-\frac{\mu_{2} b_{z}^{2}}{4 \pi d} \\
& F_{\theta}^{(2)}(d, \alpha)=\frac{\mu_{2} b_{z}^{2} n}{4 \pi d}\left(\frac{\cos n \alpha}{\sin n \alpha}+k \frac{\sin n \alpha}{\cos n \alpha}\right)
\end{aligned}
$$

for material 2. It is shown in Eqs. (73a) and (74a) that the radial image force $F_{r}^{(1)}$ in material 1 is independent on the apex angle and circumferential location $\alpha$ of the screw dislocation. Both image forces, $F_{r}^{(1)}$ and $F_{\theta}^{(1)}$, are proportional to $1 / d$. It is interesting to note that the image force $F_{r}^{(1)}$ is always negative in the wedge, the image force $F_{\theta}^{(1)}$ is zero along $\alpha=\left(\tan ^{-1} \sqrt{1 / k}\right) / n$ for $k>0$ (i.e. $\left.\mu_{1}>\mu_{2}\right)$. However, the image 
force $F_{\theta}^{(1)}$ is non-zero in the wedge for the case $k<0$. Similar features occur for the screw dislocation located in material 2.

\subsubsection{Fixed-fixed boundary condition}

From Eqs. (31), (71) and (72), the image forces exerted on a screw dislocation located at $(d, \alpha)$ for the fixed boundary condition are

$$
\begin{aligned}
& F_{r}^{(1)}(d, \alpha)=\frac{\mu_{1} b_{z}^{2}}{4 \pi d}(2 n(1-k)-1) \\
& F_{\theta}^{(1)}(d, \alpha)=\frac{n \mu_{1} b_{z}^{2}}{4 \pi d}\left(\frac{\cos n \alpha}{\sin n \alpha}+k \frac{\sin n \alpha}{\cos n \alpha}\right)
\end{aligned}
$$

for material 1 , and

$$
\begin{aligned}
& F_{r}^{(2)}(d, \alpha)=-\frac{\mu_{2} b_{z}^{2}}{4 \pi d}(1-2 n(1+k)) \\
& F_{\theta}^{(2)}(d, \alpha)=-\frac{n \mu_{2} b_{z}^{2}}{4 \pi d}\left(\frac{\cos n \alpha}{\sin n \alpha}-k \frac{\sin n \alpha}{\cos n \alpha}\right)
\end{aligned}
$$

for material 2. It is quite different from traction free boundary that the radial image force $F_{r}$ for the fixed boundary condition is dependent on the apex angle and the material constant of material 2 . The image force $F_{r}^{(1)}$ is zero in the situation that $2 n(1-k)=1$ for $k>0$ (i.e. $\mu_{1}>\mu_{2}$ ). However, the image force $F_{r}^{(1)}$ is non-zero in the wedge for the case $k<0$.

\subsection{Image forces exerted on screw dislocations for composite wedges with finite radius}

\subsubsection{Free-free-free boundary condition}

From Eqs. (37), (B.2), (71) and (72), the image forces exerted on screw dislocations for traction free boundary condition are

$$
\begin{aligned}
F_{r}^{(1)}(d, \alpha)= & -\frac{\mu_{1} b_{z}^{2}}{2 \pi d}\left[\frac{1}{2}+\frac{n(d / b)^{2 n}\left((d / b)^{2 n}+1\right)(1-\cos 2 n \alpha)}{\left((d / b)^{2 n}-1\right)\left((d / b)^{4 n}-2(d / b)^{2 n} \cos 2 n \alpha+1\right)}\right. \\
& \left.+\frac{n k(d / b)^{2 n}\left(1-(d / b)^{2 n}\right)(1-\cos 2 n \alpha)}{\left((d / b)^{2 n}+1\right)\left((d / b)^{4 n}+2(d / b)^{2 n} \cos 2 n \alpha+1\right)}\right] \\
F_{\theta}^{(1)}(d, \alpha)= & \frac{n \mu_{1} b_{z}^{2}}{2 \pi d}\left[-\frac{1}{2} \frac{\cos n \alpha}{\sin n \alpha}+\frac{(d / b)^{2 n} \sin 2 n \alpha}{(d / b)^{4 n}-2(d / b)^{2 n} \cos 2 n \alpha+1}+\frac{k}{2} \frac{\sin n \alpha}{\cos n \alpha}\right. \\
& \left.-\frac{k(d / b)^{2 n} \sin 2 n \alpha}{(d / b)^{4 n}+2(d / b)^{2 n} \cos 2 n \alpha+1}\right]
\end{aligned}
$$

for material 1 , and 


$$
\begin{aligned}
F_{r}^{(2)}(d, \alpha)= & -\frac{\mu_{2} b_{z}^{2}}{2 \pi d}\left[\frac{1}{2}+\frac{n(d / b)^{2 n}\left((d / b)^{2 n}+1\right)(1-\cos 2 n \alpha)}{\left((d / b)^{2 n}-1\right)\left((d / b)^{4 n}-2(d / b)^{2 n} \cos 2 n \alpha+1\right)}\right. \\
& \left.-\frac{n k(d / b)^{2 n}\left(1-(d / b)^{2 n}\right)(1-\cos 2 n \alpha)}{\left((d / b)^{2 n}+1\right)\left((d / b)^{4 n}+2(d / b)^{2 n} \cos 2 n \alpha+1\right)}\right] \\
F_{\theta}^{(2)}(d, \alpha)= & \frac{n \mu_{2} b_{z}^{2}}{2 \pi d}\left[\frac{1}{2} \frac{\cos n \alpha}{\sin n \alpha}-\frac{(d / b)^{2 n} \sin 2 n \alpha}{(d / b)^{4 n}-2(d / b)^{2 n} \cos 2 n \alpha+1}+\frac{k}{2} \frac{\sin n \alpha}{\cos n \alpha}\right. \\
& \left.-\frac{k(d / b)^{2 n} \sin 2 n \alpha}{(d / b)^{4 n}+2(d / b)^{2 n} \cos 2 n \alpha+1}\right]
\end{aligned}
$$

for material 2. The first term in Eqs. (77a) and (78a) represents the image force induced by the radial edges while the second and third terms in (77a) and (78a) are image forces induced by the circular boundary $r=b$.

\subsubsection{Fixed-fixed-free boundary condition}

From Eqs. (45), (B.6), (71) and (72), the image forces exerted on screw dislocations with two fixed and one traction free boundary conditions are

$$
\begin{aligned}
F_{r}^{(1)}(d, \alpha)= & \frac{\mu_{1} b_{z}^{2}}{2 \pi d}\left[n(1-k)-\frac{1}{2}+\frac{n(d / b)^{2 n}\left(\left(3(d / b)^{2 n}-1\right) \cos 2 n \alpha-2(d / b)^{4 n}+(d / b)^{2 n}-1\right)}{\left((d / b)^{2 n}-1\right)\left((d / b)^{4 n}-2(d / b)^{2 n} \cos 2 n \alpha+1\right)}\right. \\
& \left.+\frac{n k(d / b)^{2 n}\left(\left(3(d / b)^{2 n}+1\right) \cos 2 n \alpha+2(d / b)^{4 n}+(d / b)^{2 n}+1\right)}{\left((d / b)^{2 n}+1\right)\left((d / b)^{4 n}+2(d / b)^{2 n} \cos 2 n \alpha+1\right)}\right] \\
F_{\theta}^{(1)}(d, \alpha)= & \frac{n \mu_{1} b_{z}^{2}}{2 \pi d}\left[\frac{1}{2} \frac{\cos n \alpha}{\sin n \alpha}-\frac{(d / b)^{2 n} \sin 2 n \alpha}{(d / b)^{4 n}-2(d / b)^{2 n} \cos 2 n \alpha+1}+\frac{k}{2} \frac{\sin n \alpha}{\cos n \alpha}\right. \\
& \left.-\frac{k(d / b)^{2 n} \sin 2 n \alpha}{(d / b)^{4 n}+2(d / b)^{2 n} \cos 2 n \alpha+1}\right]
\end{aligned}
$$

for material 1 , and

$$
\begin{aligned}
F_{r}^{(2)}(d, \alpha)= & \frac{\mu_{2} b_{z}^{2}}{2 \pi d}\left[n(1+k)-\frac{1}{2}+\frac{n(d / b)^{2 n}\left(\left(3(d / b)^{2 n}-1\right) \cos 2 n \alpha-2(d / b)^{4 n}+(d / b)^{2 n}-1\right)}{\left((d / b)^{2 n}-1\right)\left((d / b)^{4 n}-2(d / b)^{2 n} \cos 2 n \alpha+1\right)}\right. \\
& \left.-\frac{n k(d / b)^{2 n}\left(\left(3(d / b)^{2 n}+1\right) \cos 2 n \alpha+2(d / b)^{4 n}+(d / b)^{2 n}+1\right)}{\left((d / b)^{2 n}+1\right)\left((d / b)^{4 n}+2(d / b)^{2 n} \cos 2 n \alpha+1\right)}\right] \\
F_{\theta}^{(2)}(d, \alpha)= & \frac{n \mu_{2} b_{z}^{2}}{2 \pi d}\left[-\frac{1}{2} \frac{\cos n \alpha}{\sin n \alpha}+\frac{(d / b)^{2 n} \sin 2 n \alpha}{(d / b)^{4 n}-2(d / b)^{2 n} \cos 2 n \alpha+1}+\frac{k}{2} \frac{\sin n \alpha}{\cos n \alpha}\right. \\
& \left.-\frac{k(d / b)^{2 n} \sin 2 n \alpha}{(d / b)^{4 n}+2(d / b)^{2 n} \cos 2 n \alpha+1}\right]
\end{aligned}
$$

for material 2. 


\subsection{Image force exerted on screw dislocations for annular composite wedges}

\subsubsection{Free-free-free-free boundary condition}

From Eqs. (58), (D.2), (71) and (72), the image forces exerted on screw dislocations for annular composite wedges with traction free boundary condition can be expressed as

$$
\begin{aligned}
& F_{r}^{(1)}(d, \alpha)=-\frac{\mu_{1} b_{z}^{2}}{4 \pi d}-\frac{n \mu_{1} b_{z}^{2}}{2 \pi d} \sum_{\ell=0}^{\infty} \sum_{j=1}^{4}(-1)^{j}\left[\Gamma^{-}\left(r_{j}, 0\right)-\Gamma^{-}\left(r_{j}, 2 n \alpha\right)+k \Gamma^{+}\left(r_{j}, 0\right)-k \Gamma^{+}\left(r_{j}, 2 n \alpha\right)\right] \\
& F_{\theta}^{(1)}(d, \alpha)=-\frac{n \mu_{1} b_{z}^{2}}{4 \pi d}\left(\frac{\cos n \alpha}{\sin n \alpha}-k \frac{\sin n \alpha}{\cos n \alpha}\right)+\frac{n \mu_{1} b_{z}^{2}}{2 \pi d} \sum_{\ell=0}^{\infty} \sum_{j=1}^{4}(-1)^{j}\left[\Theta^{-}\left(r_{j}, 2 n \alpha\right)-k \Theta^{+}\left(r_{j}, 2 n \alpha\right)\right]
\end{aligned}
$$

for material 1 , and

$$
\begin{aligned}
F_{r}^{(2)}(d, \alpha)= & -\frac{\mu_{2} b_{z}^{2}}{4 \pi d}-\frac{n \mu_{2} b_{z}^{2}}{2 \pi d} \sum_{\ell=0}^{\infty} \sum_{j=1}^{4}(-1)^{j}\left[\Gamma^{-}\left(r_{j}, 0\right)-\Gamma^{-}\left(r_{j}, 2 \pi-2 n \alpha\right)-k \Gamma^{+}\left(r_{j}, 0\right)\right. \\
& \left.+k \Gamma^{+}\left(r_{j}, 2 \pi-2 n \alpha\right)\right] \\
F_{\theta}^{(2)}(d, \alpha)= & \frac{\mu_{2} b_{z}^{2}}{4 \pi d}\left(\frac{\cos n \alpha}{\sin n \alpha}+k \frac{\sin n \alpha}{\cos n \alpha}\right)+\frac{n \mu_{2} b_{z}^{2}}{2 \pi d} \sum_{\ell=0}^{\infty} \sum_{j=1}^{4}(-1)^{j}\left[\Theta^{-}\left(r_{j}, 2 \pi-2 n \alpha\right)-k \Theta^{+}\left(r_{j}, 2 \pi-2 n \alpha\right)\right]
\end{aligned}
$$

for material 2, where

$$
r_{1}=(b / a)^{2 n(\ell+1)}, \quad r_{2}=\left((b / a)^{2(\ell+1)}(d / b)^{2}\right)^{n}, \quad r_{3}=(a / b)^{2 n(\ell+1)}, \quad r_{4}=\left((a / b)^{2 \ell}(d / b)^{2}\right)^{n}
$$

The second term with summation represented in Eqs. (81) and (82) are image forces exerted on screw dislocations by circular boundaries at $r=a$ and $r=b$.

\subsubsection{Fixed-fixed-free-fixed boundary condition}

From Eqs. (69), (D.6), (71) and (72), the image forces exerted on screw dislocations for annular composite wedges with three fixed and one traction free boundary conditions are

$$
\begin{aligned}
F_{r}^{(1)}(d, \alpha)= & \frac{\mu_{1} b_{z}^{2}}{4 \pi d}(2 n(1-k)-1)-\frac{n \mu_{1} b_{z}^{2}}{2 \pi d} \sum_{\ell=0}^{\infty} \sum_{j=1}^{4}(-1)^{\ell+j}\left[\Gamma^{-}\left(r_{j}, 0\right)+\Gamma^{-}\left(r_{j}, 2 n \alpha\right)\right. \\
& \left.-k \Gamma^{+}\left(r_{j}, 0\right)-k \Gamma^{+}\left(r_{j}, 2 n \alpha\right)\right] \\
F_{\theta}^{(1)}(d, \alpha)= & \frac{n \mu_{1} b_{z}^{2}}{4 \pi d}\left(\frac{\cos n \alpha}{\sin \alpha}+k \frac{\sin n \alpha}{\cos n \alpha}\right)-\frac{n \mu_{1} b_{z}^{2}}{2 \pi d} \sum_{\ell=0}^{\infty} \sum_{j=1}^{4}(-1)^{\ell+j}\left[\Theta^{-}\left(r_{j}, 2 n \alpha\right)+k \Theta^{+}\left(r_{j}, 2 n \alpha\right)\right]
\end{aligned}
$$

for material 1 , and

$$
\begin{aligned}
F_{r}^{(2)}(d, \alpha)= & \frac{\mu_{2} b_{z}^{2}}{4 \pi d}(2 n(1+k)-1)-\frac{n \mu_{2} b_{z}^{2}}{2 \pi d} \sum_{\ell=0}^{\infty} \sum_{j=1}^{4}(-1)^{\ell+j}\left[\Gamma^{-}\left(r_{j}, 0\right)+\Gamma^{-}\left(r_{j}, 2 \pi-2 n \alpha\right)\right. \\
& \left.+k \Gamma^{+}\left(r_{j}, 0\right)+k \Gamma^{+}\left(r_{j}, 2 \pi-2 n \alpha\right)\right] \\
F_{\theta}^{(2)}(d, \alpha)= & \frac{n \mu_{2} b_{z}^{2}}{4 \pi d}\left(-\frac{\cos n \alpha}{\sin n \alpha}+k \frac{\sin n \alpha}{\cos n \alpha}\right)-\frac{n \mu_{2} b_{z}^{2}}{2 \pi d} \sum_{\ell=0}^{\infty} \sum_{j=1}^{4}(-1)^{\ell+j}\left[\Theta^{-}\left(r_{j}, 2 \pi-2 n \alpha\right)+k \Theta^{+}\left(r_{j}, 2 \pi-2 n \alpha\right)\right]
\end{aligned}
$$


for material 2, where

$$
r_{1}=(b / a)^{2 n(\ell+1)}, \quad r_{2}=\left((b / a)^{2(\ell+1)}(d / b)^{2}\right)^{n}, \quad r_{3}=\left((a / b)^{2 \ell}(d / b)^{2}\right)^{n}, \quad r_{4}=(a / b)^{2 n(\ell+1)}
$$

The second term with summation presented in Eqs. (84) and (85) are image forces exerted on screw dislocations by circular boundaries at $r=a$ and $r=b$.

From the available solutions of full-field stresses presented in this study, it is easy to construct the image forces exerted on screw dislocations in analytical forms. Although we only present the results for two different boundary conditions for each case in this section, the image forces exerted on screw dislocations for other boundary conditions can be obtained without difficulty.

\section{Numerical results of full-field stress distributions and image forces}

The analytical full-field solutions of shear stresses for various boundary conditions are explicitly presented in Section 4 for composite sharp wedges with finite radius and in Section 5 for composite annular wedges. The solutions are functions of wedge angle $\beta$, finite radii $a$ and $b$, material constants $\mu_{1}$ and $\mu_{2}$, and the location of the concentrated load (or screw dislocation) $(d, \alpha)$. The full-field stress distributions for composite wedges with a finite radius subjected to concentrated loads are calculated and shown in Figs. 8-10
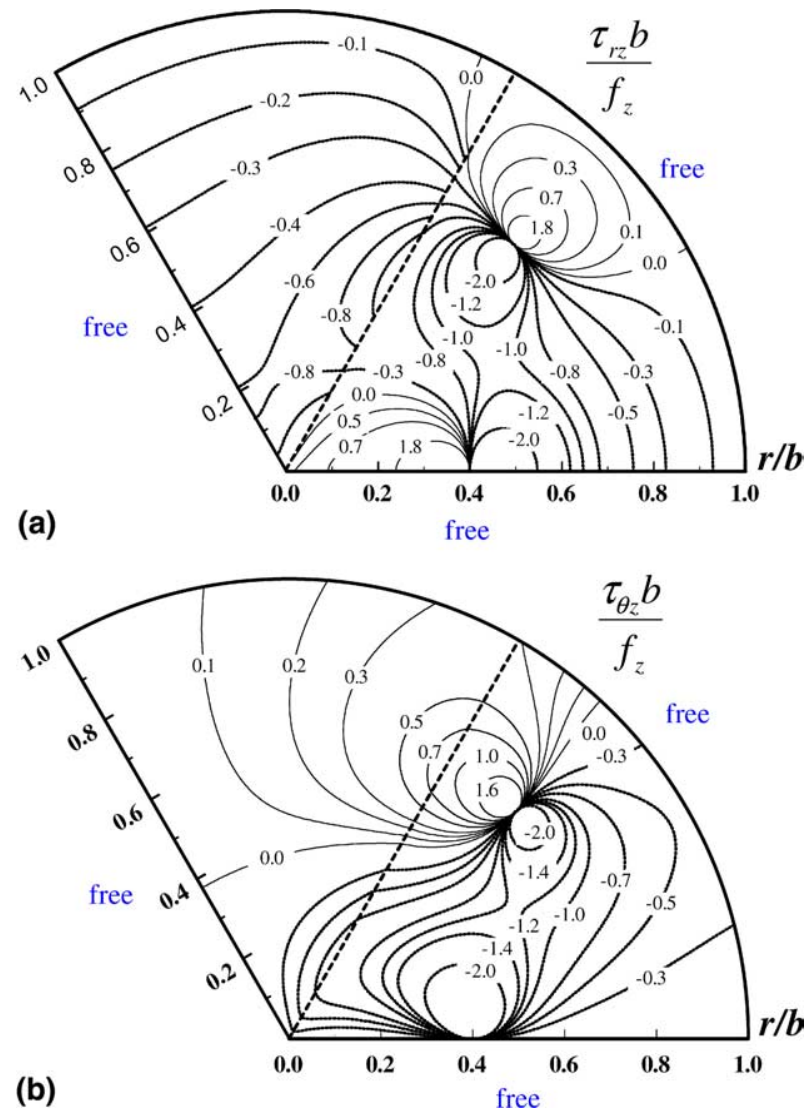

Fig. 8. (a) The full-field distribution of shear stress $\tau_{r z}$ for a composite sharp wedge with an apex angle $\beta=120^{\circ}$ subjected to selfequilibrium forces located at $\left(0.4 b, 0^{\circ}\right)$ and $\left(0.7 b, 45^{\circ}\right)$. (b) The full-field distribution of shear stress $\tau_{\theta z}$ for a composite sharp wedge with an apex angle $\beta=120^{\circ}$ subjected to self-equilibrium forces located at $\left(0.4 b, 0^{\circ}\right)$ and $\left(0.7 b, 45^{\circ}\right)$. 
for wedge angles $\beta=120^{\circ}$ and the ratio of shear modulus for materials 1 and 2 is $\mu_{1} / \mu_{2}=1 / 2$. Figs. $8-10$ present the shear stresses for three different boundary conditions. It is indicated in these figures that the traction free boundary condition along the two edges for $\tau_{\theta z}$ and along the circular segment for $\tau_{r z}$ are satisfied. The continuity condition along the interface for stress $\tau_{\theta z}$ is satisfied while $\tau_{r z}$ is discontinuous along the interface. Because $\beta=120^{\circ}<180^{\circ}$, no stress singularities near the apex of the composite wedge are found for Figs. 8-10. The full-field distributions of shear stresses for composite wedges with a finite radius subjected to a screw dislocation is shown in Fig. 11 for a wedge angle $\beta=120^{\circ}$. The boundary conditions for Fig. 11 are fixed along two edges and traction free along the circular segment, and $\mu_{1} / \mu_{2}=1 / 2$. Figs. $12-14$ present the shear stresses for a composite wedge with largest apex angle $\beta=360^{\circ}$ which is the problem of a composite disk with an interface crack. In this case, the apex of the composite wedge is equivalent to an interface crack tip and the well known square root stress singularities in the crack tip are clearly presented in these figures. Figs. 15-17 are stress distributions of composite annular wedges subjected to concentrated loads for three different boundary conditions indicated in Eqs. (53), (61) and (65), respectively. The apex angle and radial length of the composite annular wedge is $\beta=120^{\circ}$ and $a=0.5 b$. The ratio of shear modulus for materials 1 and 2 is $\mu_{1} / \mu_{2}=2$. It is worthy to note that the stresses distributions satisfy all the corresponding boundary and continuity conditions. Figs. 18 and 19 are stress distributions of composite annular
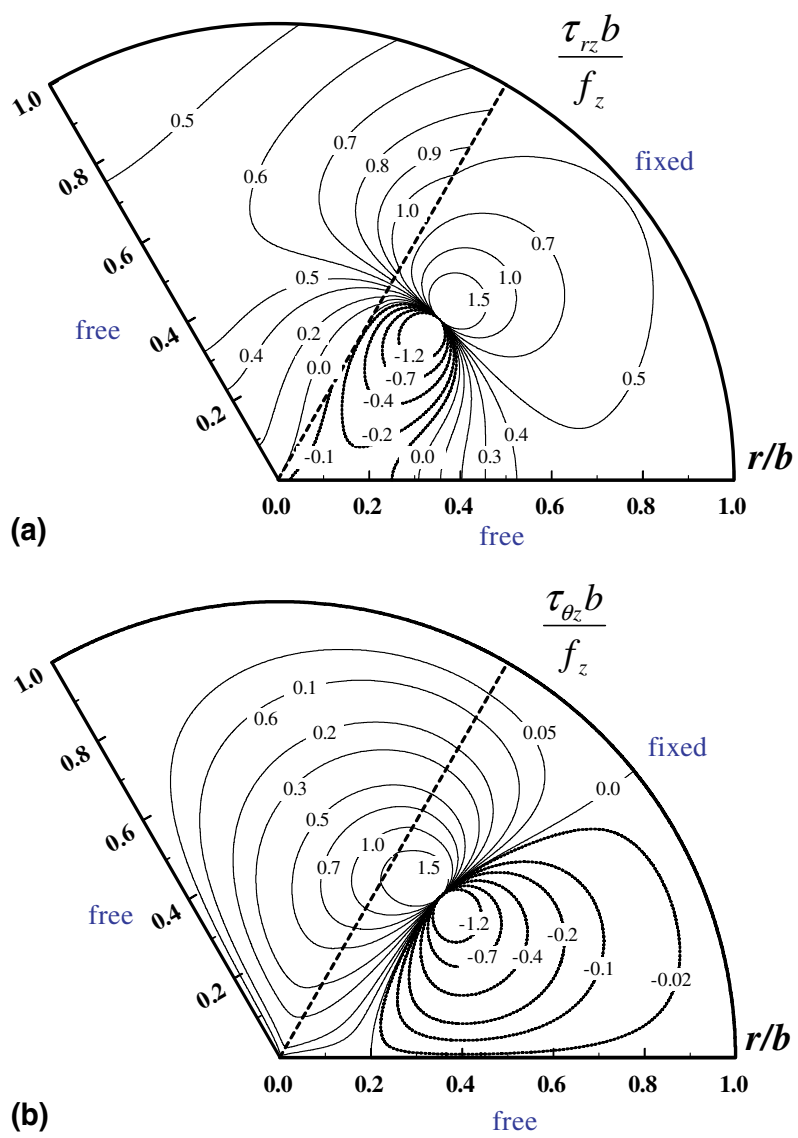

Fig. 9. (a) The full-field distribution of shear stress $\tau_{r z}$ for a composite sharp wedge with an apex angle $\beta=120^{\circ}$ subjected to a concentrated force located at $\left(0.5 b, 45^{\circ}\right)$. (b) The full-field distribution of shear stress $\tau_{\theta z}$ for a composite sharp wedge with an apex angle $\beta=120^{\circ}$ subjected to a concentrated force located at $\left(0.5 b, 45^{\circ}\right)$. 

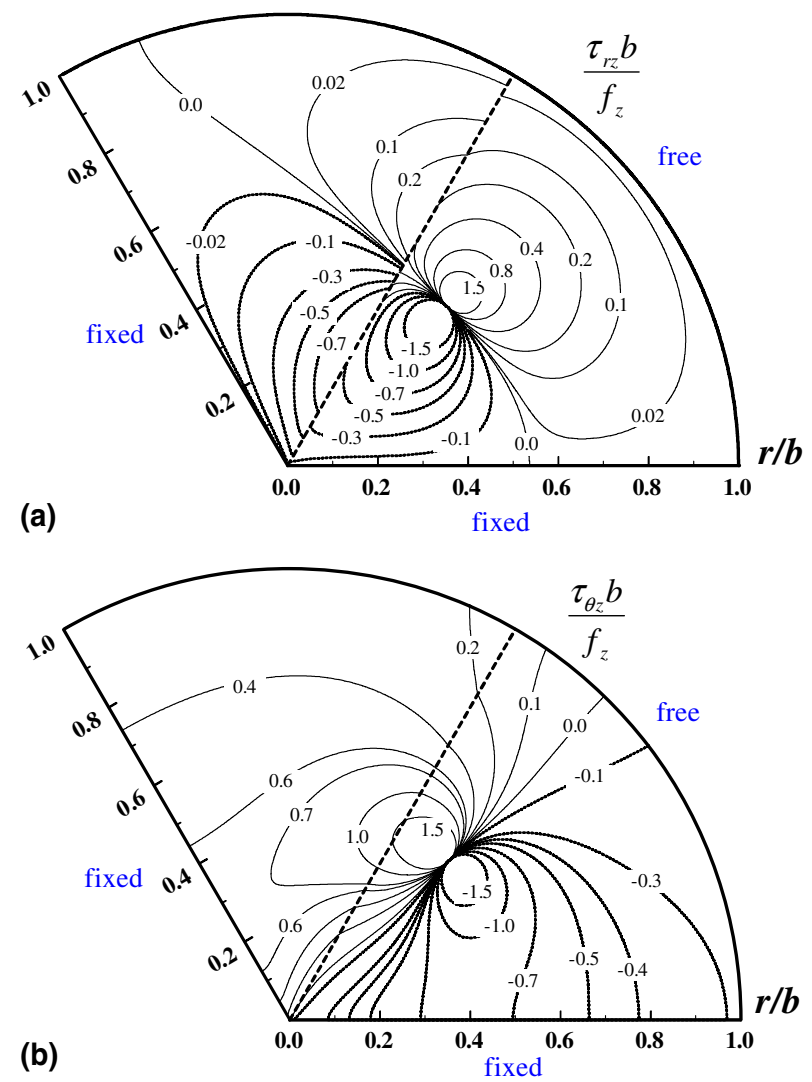

Fig. 10. (a) The full-field distribution of shear stress $\tau_{r z}$ for a composite sharp wedge with an apex angle $\beta=120^{\circ}$ subjected to a concentrated force located at $\left(0.5 b, 45^{\circ}\right)$. (b) The full-field distribution of shear stress $\tau_{\theta z}$ for a composite sharp wedge with an apex angle $\beta=120^{\circ}$ subjected to a concentrated force located at $\left(0.5 b, 45^{\circ}\right)$.

wedges subjected to a screw dislocation for two different boundary conditions. The apex angle and radial length of the composite annular wedge are $\beta=120^{\circ}$ and $a=0.4 b$. The ratio of shear modulus for materials 1 and 2 is $\mu_{1} / \mu_{2}=2$. It is worthy to note that the stresses distributions satisfy all the corresponding boundary and continuity conditions. The theoretical solutions of image forces $F_{r}$ and $F_{\theta}$ exerted on screw dislocations are presented in Eqs. (73)-(76) for infinite wedge, in Eqs. (77)-(80) for wedges with finite radius and in Eqs. (81)-(86) for annular wedges. The numerical results for composite wedges with finite radius are shown in Figs. 20 and 21 for $\beta=120^{\circ}$. It is found that there exist an equilibrium point (i.e. $F_{r}=F_{\theta}=0$ ) for the traction free boundary condition and the location for the equilibrium point in material 1 is indicated in Fig. 20. However, no equilibrium point is found for the case that fixed boundaries along two radial edges and free along the circular segment as shown in Fig. 21. The results of annular wedge with traction free boundary condition and apex angle $\beta=120^{\circ}$ are shown in Fig. 22. The location of the equilibrium point is also indicated in Fig. 22 which has only a slight difference to that indicated in Fig. 20.

\section{Conclusions}

In this study, a complete investigation on concentrated anti-plane forces and screw dislocations applied in isotopic composite annular wedges is presented. The explicit closed-form solutions for displacement 

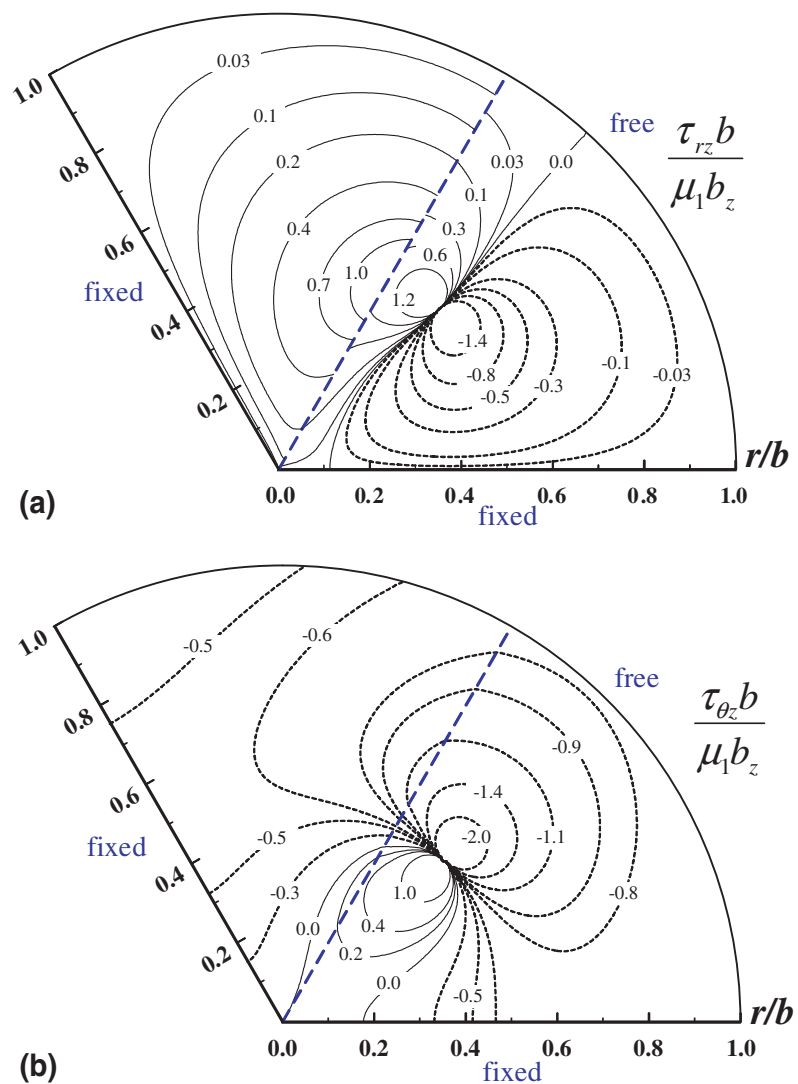

Fig. 11. (a) The full-field distribution of shear stress $\tau_{r z}$ for a composite sharp wedge with an apex angle $\beta=120^{\circ}$ subjected to a screw dislocation located at $\left(0.5 b, 45^{\circ}\right)$. (b) The full-field distribution of shear stress $\tau_{\theta z}$ for a composite sharp wedge with an apex angle $\beta=120^{\circ}$ subjected to a screw dislocation located at $\left(0.5 b, 45^{\circ}\right)$.

and shear stresses are obtained by using the Mellin transform technique and the image method. Many possible boundary conditions on radial edges and circular segments are taken into account. The special case of interface crack problem, which is intersected for many applications, is also presented in detail. It is worthy to note that even for the complicated geometrical configuration as the composite annular wedge, the full-field solution consists of only one infinite summation. Each term presented in the solution is only a combination of simple trigonometric functions. The explicit solutions with simple forms of displacement and stresses presented in this study are very easy to use for numerical investigations and theoretical analysis. The stress distributions for composite wedges with a finite radius and annular wedges are discussed from numerical calculations. The image forces exerted on screw dislocations are derived and the equilibrium points are identified base on the numerical calculations for special boundary conditions.

\section{Acknowledgements}

The financial support of the authors from the National Science Council, Republic of China, through Grant NSC 90-2212-E002-230 to National Taiwan University is gratefully acknowledged. 

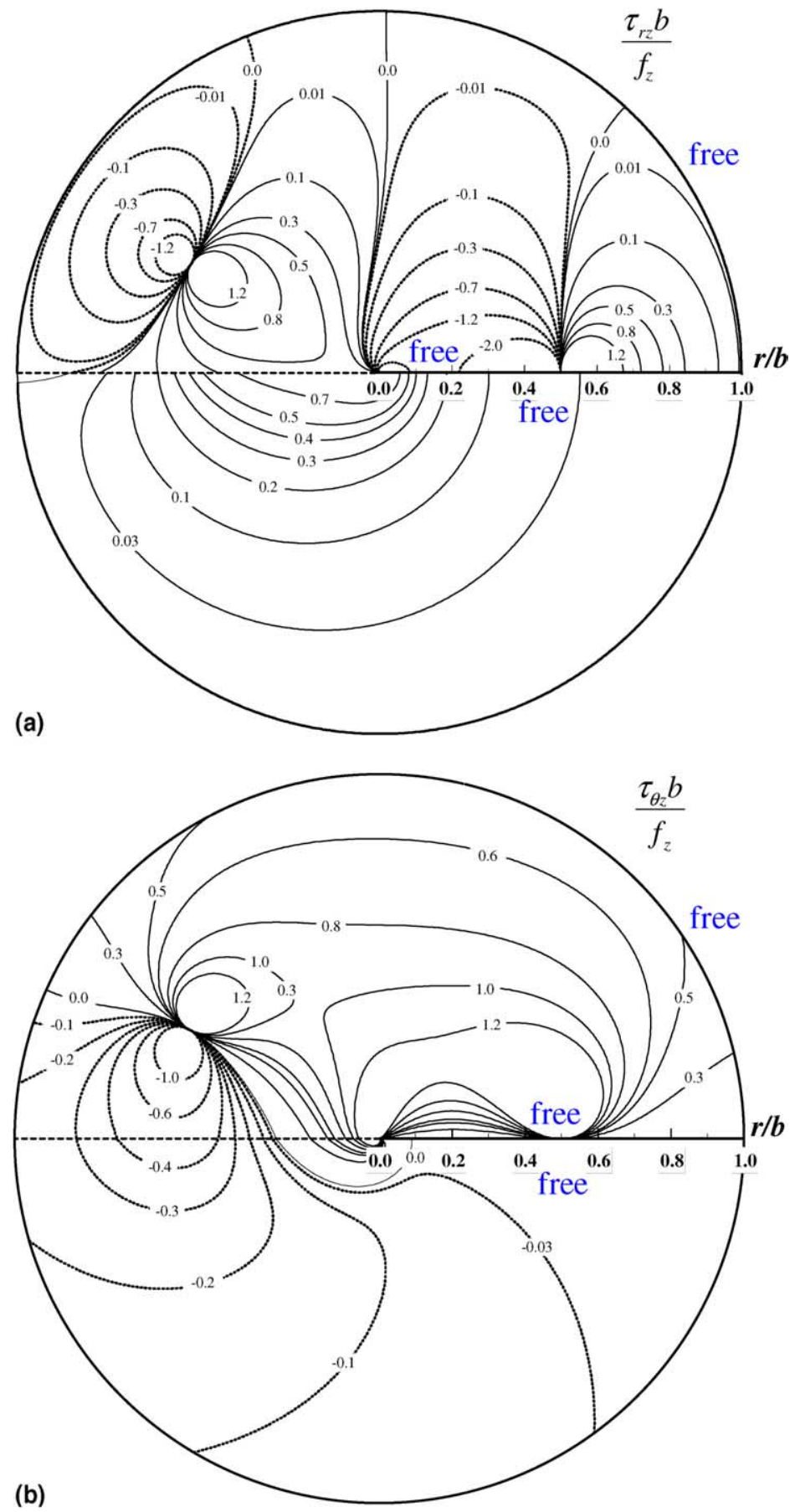

Fig. 12. (a) The full-field distribution of shear stress $\tau_{r z}$ for a composite sharp wedge with an interface crack subjected to self-equilibrium forces located at $\left(0.5 b, 0^{\circ}\right)$ and $\left(0.6 b, 150^{\circ}\right)$. (b) The full-field distribution of shear stress $\tau_{\theta z}$ for a composite sharp wedge with an interface crack subjected to self-equilibrium forces located at $\left(0.5 b, 0^{\circ}\right)$ and $\left(0.6 b, 150^{\circ}\right)$. 

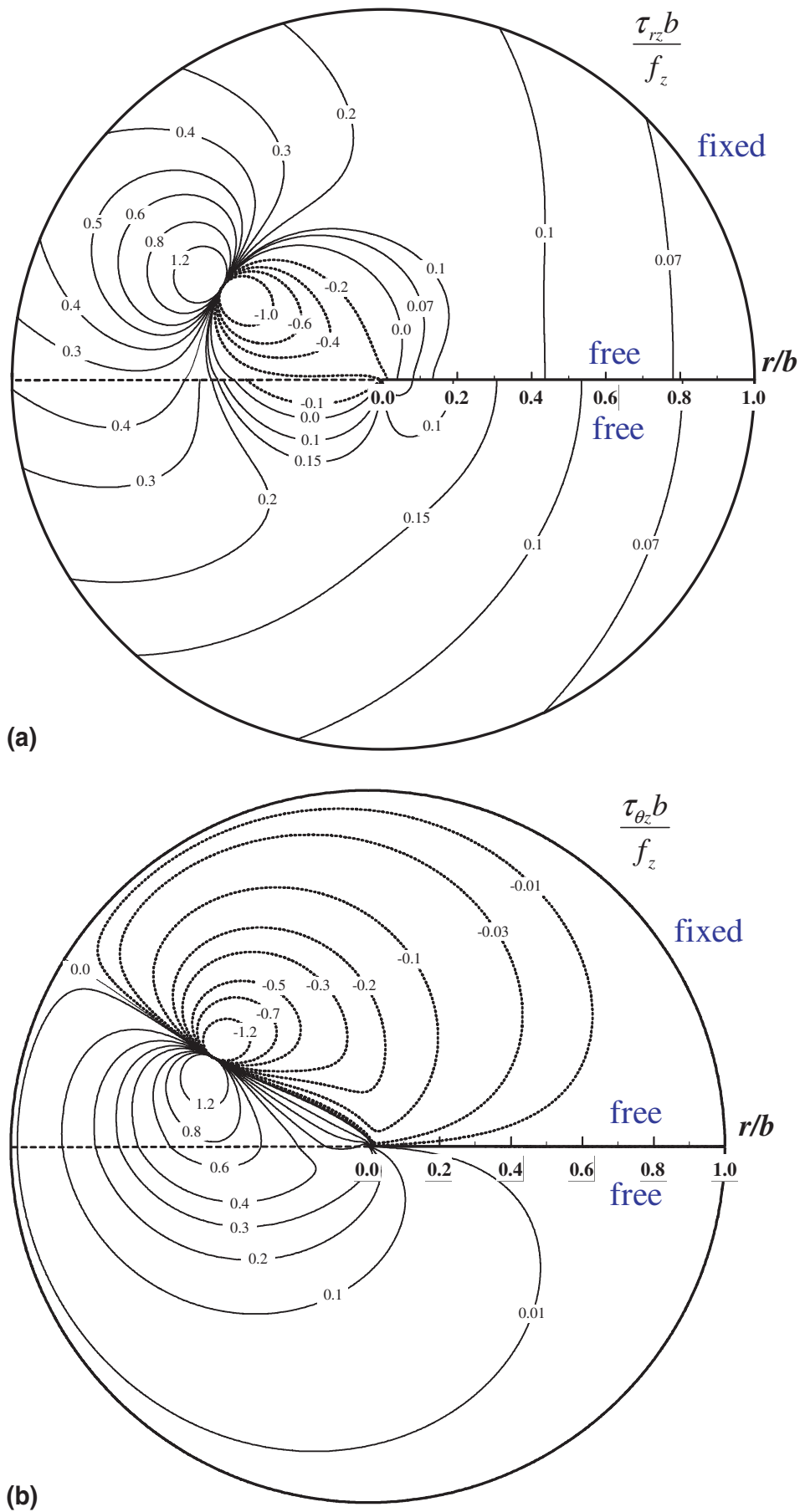

Fig. 13. (a) The full-field distribution of shear stress $\tau_{r z}$ for a composite sharp wedge with an interface crack subjected to a concentrated force located at $\left(0.5 b, 150^{\circ}\right)$. (b) The full-field distribution of shear stress $\tau_{\theta z}$ for a composite sharp wedge with an interface crack subjected to a concentrated force located at $\left(0.5 b, 150^{\circ}\right)$. 

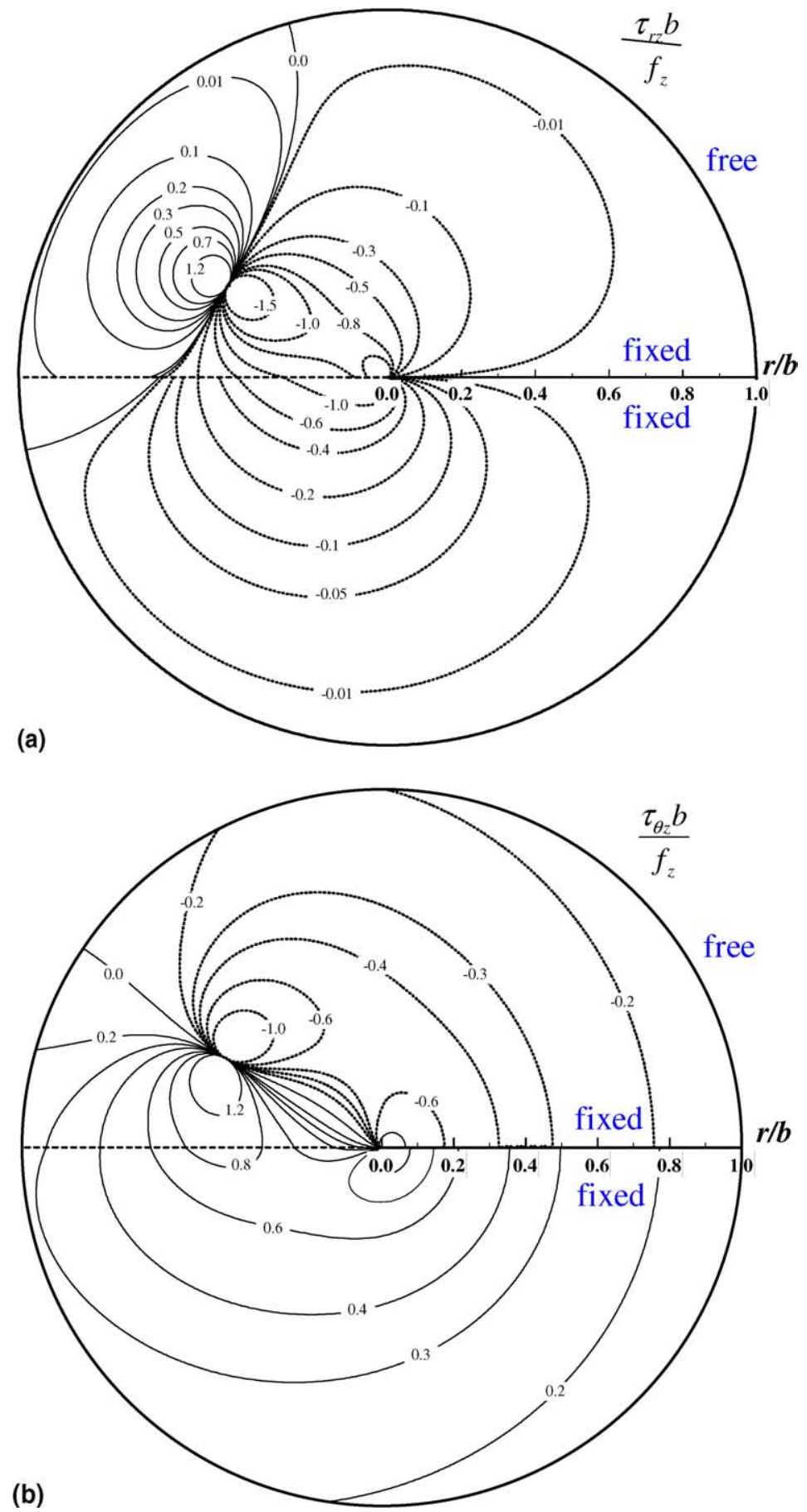

Fig. 14. (a) The full-field distribution of shear stress $\tau_{r z}$ for a composite sharp wedge with an interface crack subjected to a concentrated force located at $\left(0.5 b, 150^{\circ}\right)$. (b) The full-field distribution of shear stress $\tau_{\theta z}$ for a composite sharp wedge with an interface crack subjected to a concentrated force located at $\left(0.5 \mathrm{~b}, 150^{\circ}\right)$. 

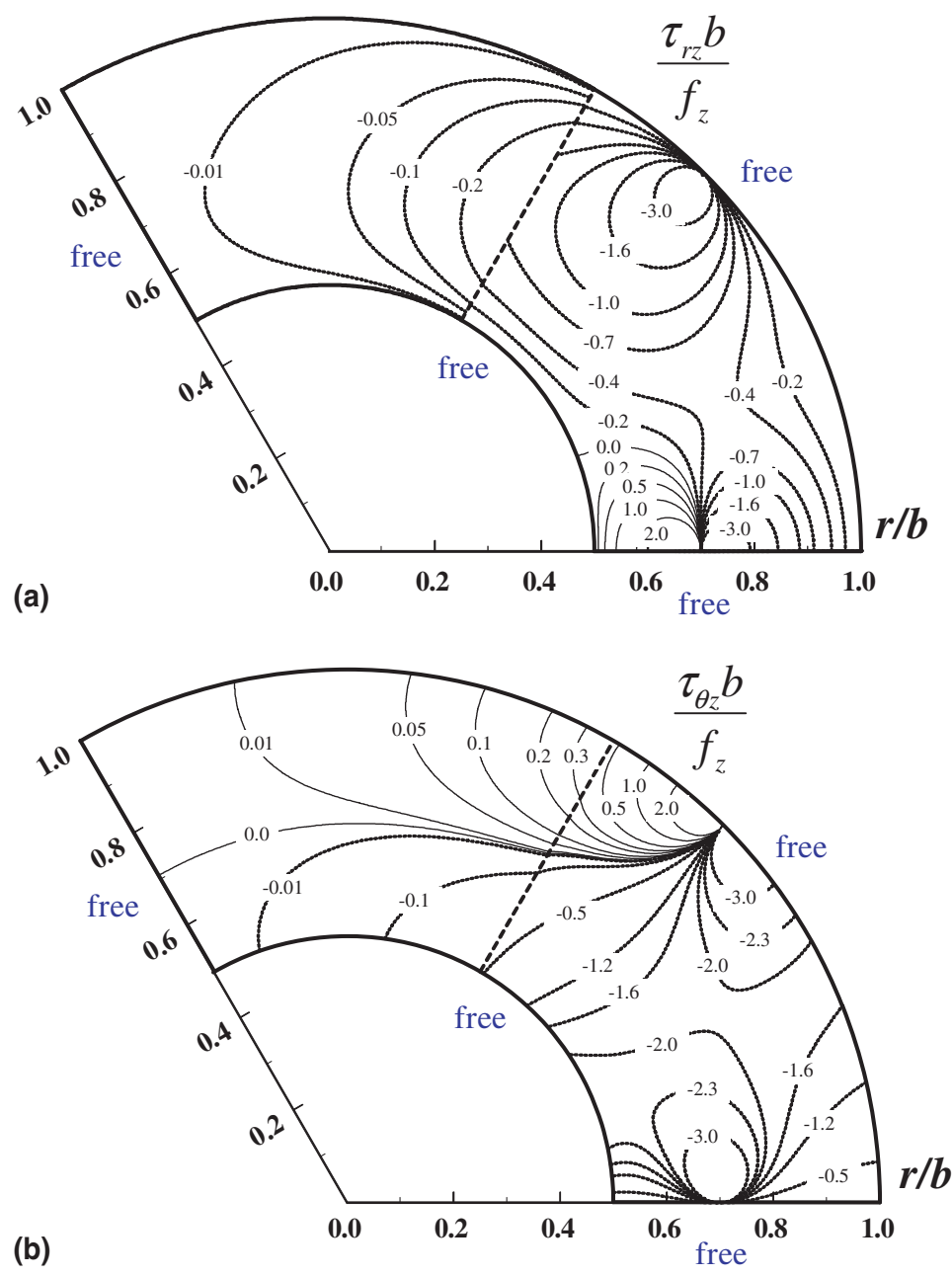

Fig. 15. (a) The full-field distribution of shear stress $\tau_{r z}$ for a composite annular wedge with an apex angle $\beta=120^{\circ}$ and $a=0.5 b$ subjected to self-equilibrium forces located at $\left(b, 45^{\circ}\right)$ and $\left(0.7 b, 0^{\circ}\right)$. (b) The full-field distribution of shear stress $\tau_{\theta z}$ for a composite annular wedge with an apex angle $\beta=120^{\circ}$ and $a=0.5 b$ subjected to self-equilibrium forces located at $\left(b, 45^{\circ}\right)$ and $\left(0.7 b, 0^{\circ}\right)$.

\section{Appendix A. The full-field solutions $w(r, \theta)$ and $\tau_{r z}(r, \theta)$ of composite sharp wedges with finite radius (concentrated loads)}

\section{A.1. Free-free-free boundary condition}

$$
\begin{aligned}
w^{(1)}= & \frac{f_{z}}{4 \pi \mu_{1}} \sum_{m=1}^{2}(-1)^{m+1}\left[\Omega^{-}\left(\left(r / d_{m}\right)^{n}, n\left(\theta-\alpha_{m}\right)\right)+\Omega^{-}\left(\left(r / d_{m}\right)^{n}, n\left(\theta+\alpha_{m}\right)\right)+k \Omega^{+}\left(\left(r / d_{m}\right)^{n}, n\left(\theta-\alpha_{m}\right)\right)\right. \\
& +k \Omega^{+}\left(\left(r / d_{m}\right)^{n}, n\left(\theta+\alpha_{m}\right)\right)+\Omega^{-}\left(\left(r d_{m} / b^{2}\right)^{n}, n\left(\theta-\alpha_{m}\right)\right)+\Omega^{-}\left(\left(r d_{m} / b^{2}\right)^{n}, n\left(\theta+\alpha_{m}\right)\right) \\
& \left.+k \Omega^{+}\left(\left(r d_{m} / b^{2}\right)^{n}, n\left(\theta-\alpha_{m}\right)\right)+k \Omega^{+}\left(\left(r d_{m} / b^{2}\right)^{n}, n\left(\theta+\alpha_{m}\right)\right)\right]
\end{aligned}
$$



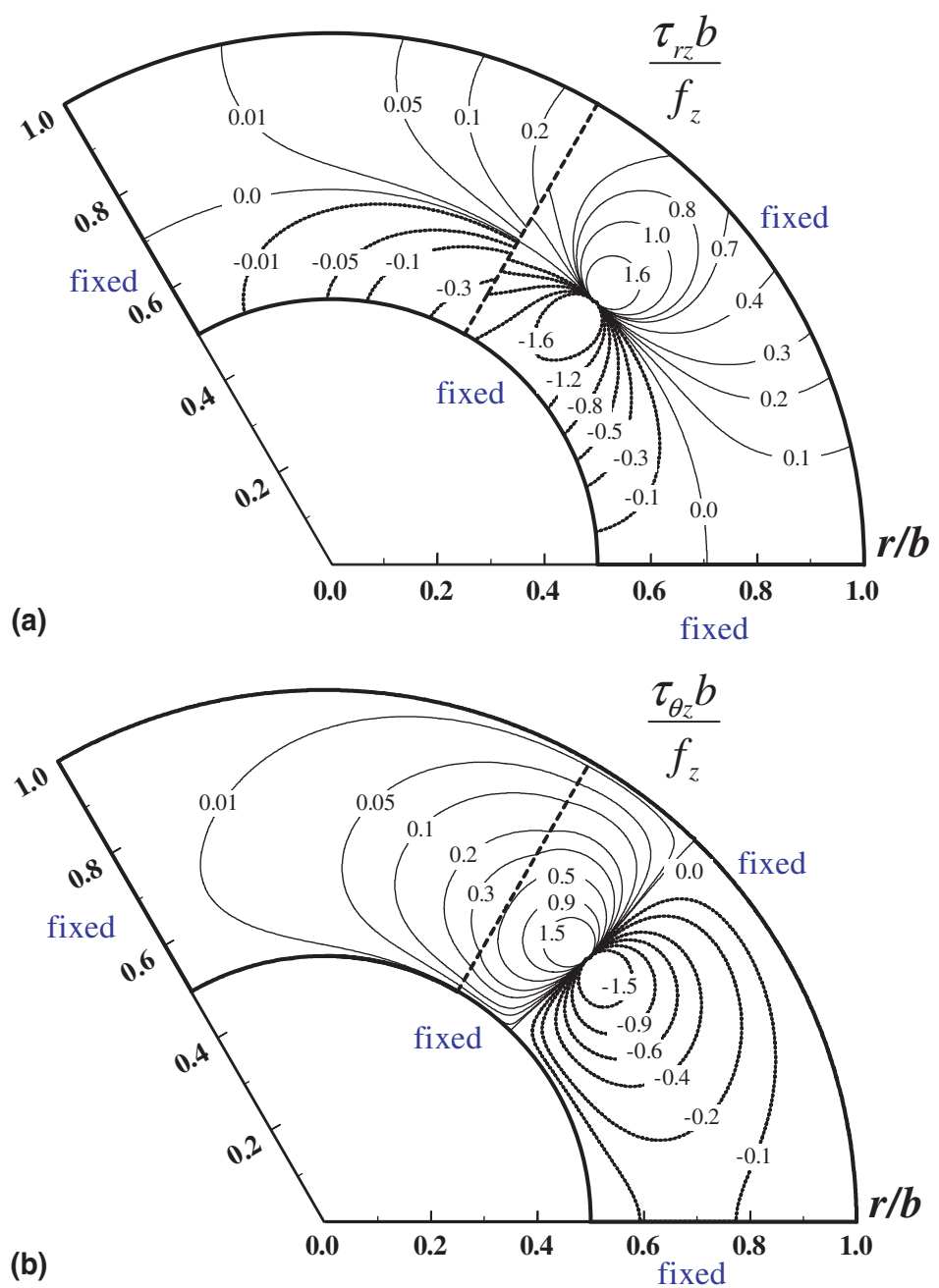

Fig. 16. (a) The full-field distribution of shear stress $\tau_{r z}$ for a composite annular wedge with an apex angle $\beta=120^{\circ}$ and $a=0.5 b$ subjected to a concentrated force located at $\left(0.8 b, 45^{\circ}\right)$. (b) The full-field distribution of shear stress $\tau_{\theta z}$ for a composite annular wedge with an apex angle $\beta=120^{\circ}$ and $a=0.5 b$ subjected to a concentrated force located at $\left(0.8 b, 45^{\circ}\right)$.

$$
\begin{aligned}
\tau_{r z}^{(1)}= & \frac{n f_{z}}{2 \pi r} \sum_{m=1}^{2}(-1)^{m+1}\left[\Gamma^{-}\left(\left(r / d_{m}\right)^{n}, n\left(\theta-\alpha_{m}\right)\right)+\Gamma^{-}\left(\left(r / d_{m}\right)^{n}, n\left(\theta+\alpha_{m}\right)\right)+k \Gamma^{+}\left(\left(r / d_{m}\right)^{n}, n\left(\theta-\alpha_{m}\right)\right)\right. \\
& +k \Gamma^{+}\left(\left(r / d_{m}\right)^{n}, n\left(\theta+\alpha_{m}\right)\right)+\Gamma^{-}\left(\left(r d_{m} / b^{2}\right)^{n}, n\left(\theta-\alpha_{m}\right)\right)+\Gamma^{-}\left(\left(r d_{m} / b^{2}\right)^{n}, n\left(\theta+\alpha_{m}\right)\right) \\
& \left.+k \Gamma^{+}\left(\left(r d_{m} / b^{2}\right)^{n}, n\left(\theta-\alpha_{m}\right)\right)+k \Gamma^{+}\left(\left(r d_{m} / b^{2}\right)^{n}, n\left(\theta+\alpha_{m}\right)\right)\right] \\
w^{(2)}= & \frac{f_{z}}{2 \pi\left(\mu_{1}+\mu_{2}\right)} \sum_{m=1}^{2}(-1)^{m+1}\left[\Omega^{-}\left(\left(r / d_{m}\right)^{n}, n\left(\theta-\alpha_{m}\right)\right)+\Omega^{-}\left(\left(r / d_{m}\right)^{n}, n\left(\theta+\alpha_{m}\right)\right)\right. \\
& \left.+\Omega^{-}\left(\left(r d_{m} / b^{2}\right)^{n}, n\left(\theta-\alpha_{m}\right)\right)+\Omega^{-}\left(\left(r d_{m} / b^{2}\right)^{n}, n\left(\theta+\alpha_{m}\right)\right)\right]
\end{aligned}
$$



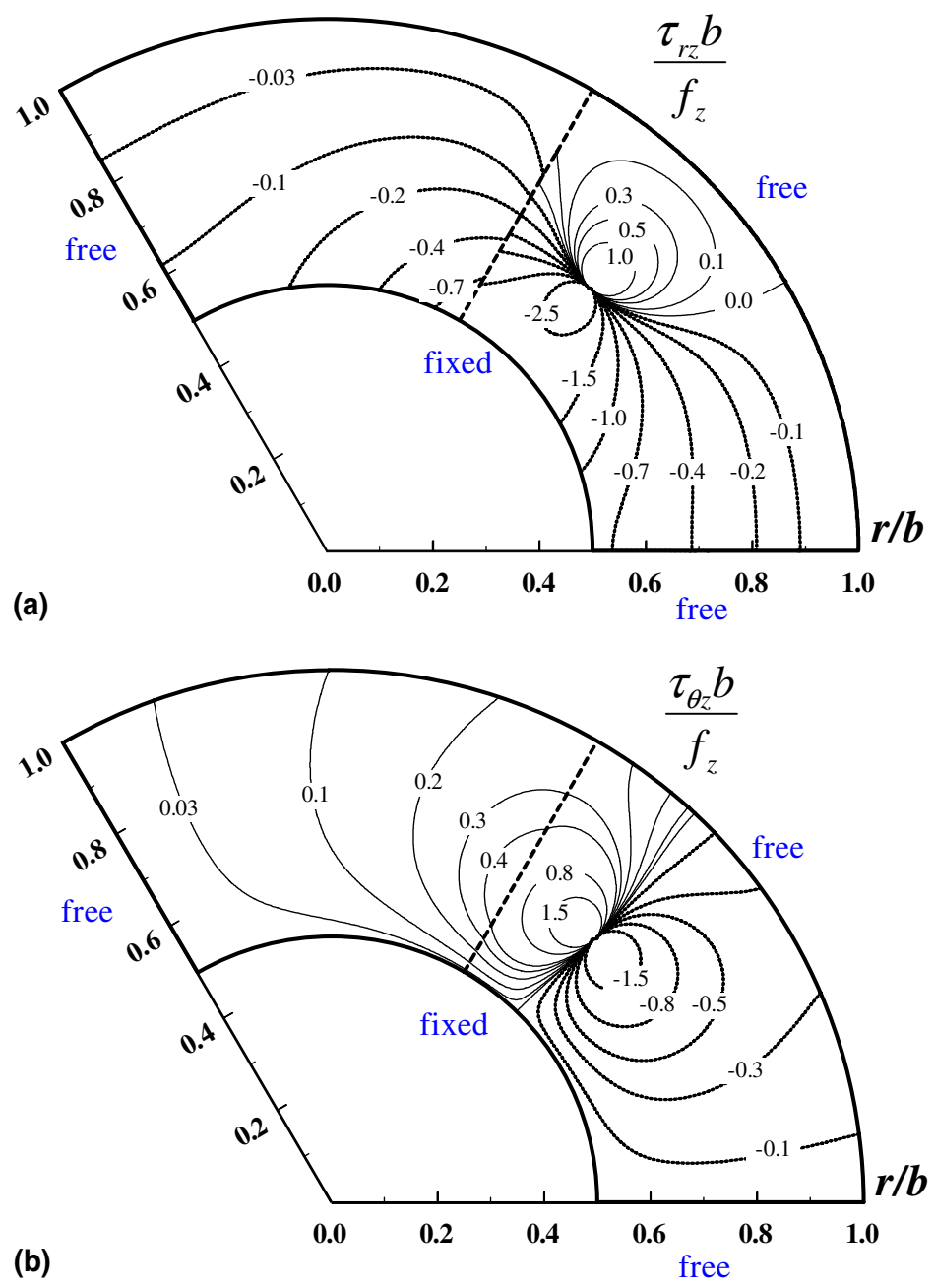

Fig. 17. (a) The full-field distribution of shear stress $\tau_{r z}$ for a composite annular wedge with an apex angle $\beta=120^{\circ}$ and $a=0.5 b$ subjected to a concentrated force located at $\left(0.8 b, 45^{\circ}\right)$. (b) The full-field distribution of shear stress $\tau_{\theta z}$ for a composite annular wedge with an apex angle $\beta=120^{\circ}$ and $a=0.5 b$ subjected to a concentrated force located at $\left(0.8 b, 45^{\circ}\right)$.

$$
\begin{aligned}
\tau_{r z}^{(2)}= & \frac{n \mu_{2} f_{z}}{\pi\left(\mu_{1}+\mu_{2}\right) r} \sum_{m=1}^{2}(-1)^{m+1}\left[\Gamma^{-}\left(\left(r / d_{m}\right)^{n}, n\left(\theta-\alpha_{m}\right)\right)+\Gamma^{-}\left(\left(r / d_{m}\right)^{n}, n\left(\theta+\alpha_{m}\right)\right)\right. \\
& \left.+\Gamma^{-}\left(\left(r d_{m} / b^{2}\right)^{n}, n\left(\theta-\alpha_{m}\right)\right)+\Gamma^{-}\left(\left(r d_{m} / b^{2}\right)^{n}, n\left(\theta+\alpha_{m}\right)\right)\right]
\end{aligned}
$$

\section{A.2. Free-free-fixed boundary condition}

$$
\begin{aligned}
w^{(1)}= & \frac{f_{z}}{4 \pi \mu_{1}}\left[\Omega^{-}\left((r / d)^{n}, n(\theta-\alpha)\right)+\Omega^{-}\left((r / d)^{n}, n(\theta+\alpha)\right)+k \Omega^{+}\left((r / d)^{n}, n(\theta-\alpha)\right)\right. \\
& +k \Omega^{+}\left((r / d)^{n}, n(\theta+\alpha)\right)-\Omega^{-}\left(\left(r d / b^{2}\right)^{n}, n(\theta-\alpha)\right)-\Omega^{-}\left(\left(r d / b^{2}\right)^{n}, n(\theta+\alpha)\right) \\
& \left.-k \Omega^{+}\left(\left(r d / b^{2}\right)^{n}, n(\theta-\alpha)\right)-k \Omega^{+}\left(\left(r d / b^{2}\right)^{n}, n(\theta+\alpha)\right)\right]
\end{aligned}
$$



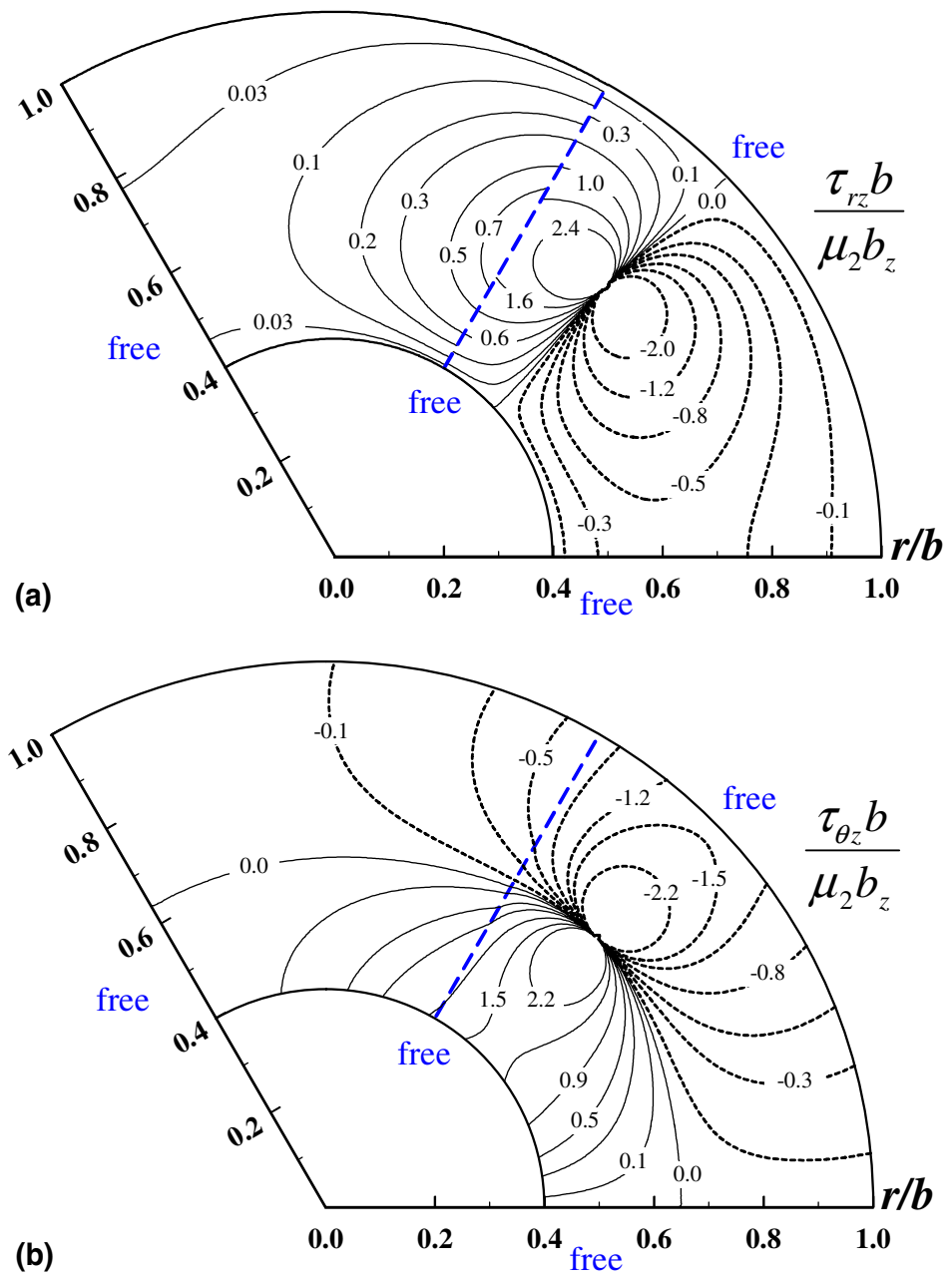

Fig. 18. (a) The full-field distribution of shear stress $\tau_{r z}$ for a composite annular wedge with an apex angle $\beta=120^{\circ}$ and $a=0.4 b$ subjected to a screw dislocation located at $\left(0.6 b, 45^{\circ}\right)$. (b) The full-field distribution of shear stress $\tau_{\theta z}$ for a composite annular wedge with an apex angle $\beta=120^{\circ}$ and $a=0.4 b$ subjected to a screw dislocation located at $\left(0.6 b, 45^{\circ}\right)$.

$$
\begin{aligned}
\tau_{r z}^{(1)}= & \frac{n f_{z}}{2 \pi r}\left[\Gamma^{-}\left((r / d)^{n}, n(\theta-\alpha)\right)+\Gamma^{-}\left((r / d)^{n}, n(\theta+\alpha)\right)+k \Gamma^{+}\left((r / d)^{n}, n(\theta-\alpha)\right)\right. \\
& +k \Gamma^{+}\left((r / d)^{n}, n(\theta+\alpha)\right)-\Gamma^{-}\left(\left(r d / b^{2}\right)^{n}, n(\theta-\alpha)\right)-\Gamma^{-}\left(\left(r d / b^{2}\right)^{n}, n(\theta+\alpha)\right) \\
& \left.-k \Gamma^{+}\left(\left(r d / b^{2}\right)^{n}, n(\theta-\alpha)\right)-k \Gamma^{+}\left(\left(r d / b^{2}\right)^{n}, n(\theta+\alpha)\right)\right] \\
w^{(2)}= & \frac{f_{z}}{2 \pi\left(\mu_{1}+\mu_{2}\right)}\left[\Omega^{-}\left((r / d)^{n}, n(\theta-\alpha)\right)+\Omega^{-}\left((r / d)^{n}, n(\theta+\alpha)\right)-\Omega^{-}\left(\left(r d / b^{2}\right)^{n}, n(\theta-\alpha)\right)\right. \\
& \left.-\Omega^{-}\left(\left(r d / b^{2}\right)^{n}, n(\theta+\alpha)\right)\right]
\end{aligned}
$$



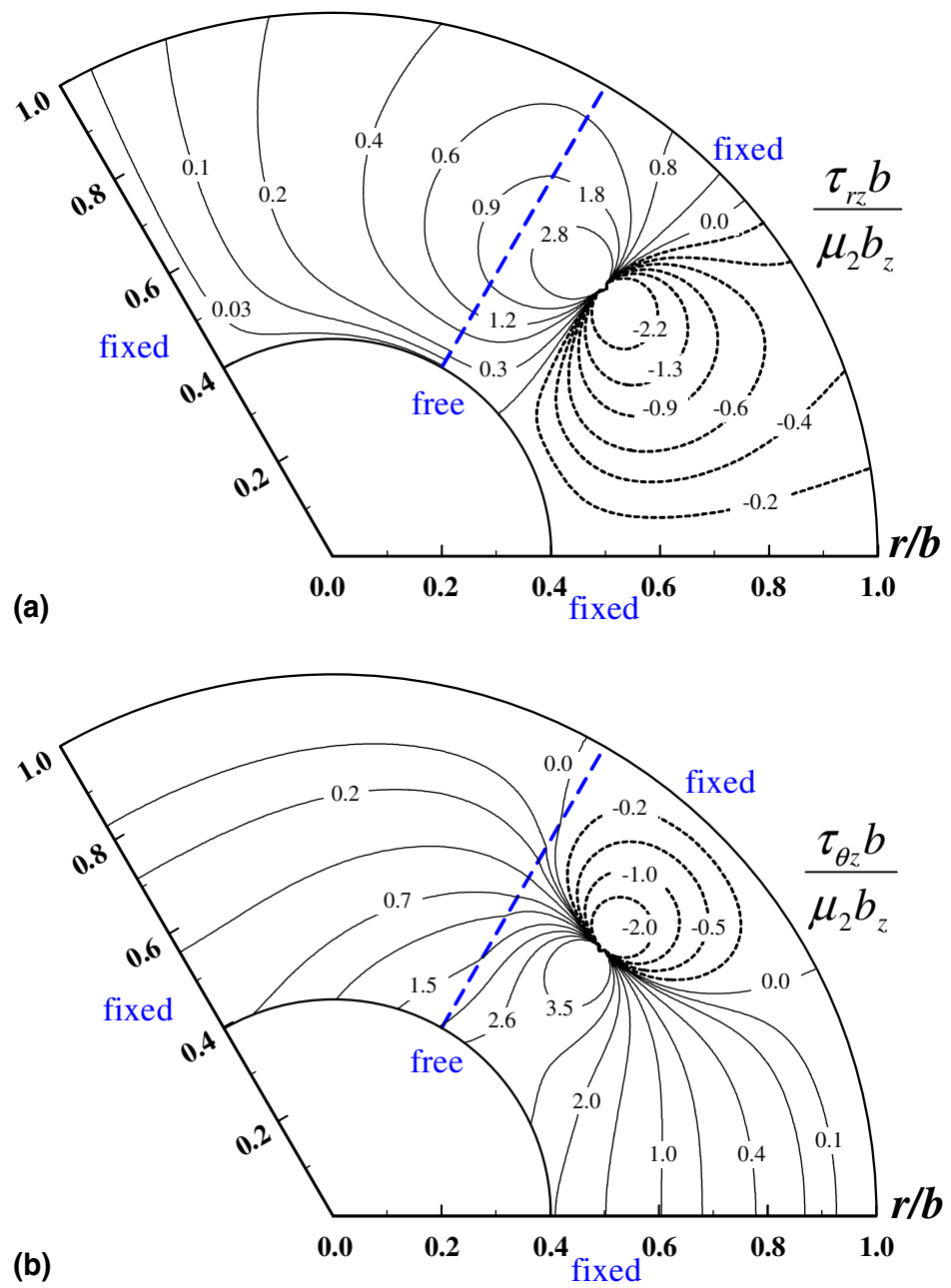

Fig. 19. (a) The full-field distribution of shear stress $\tau_{r z}$ for a composite annular wedge with an apex angle $\beta=120^{\circ}$ and $a=0.4 b$ subjected to a screw dislocation located at $\left(0.6 b, 45^{\circ}\right)$. (b) The full-field distribution of shear stress $\tau_{\theta z}$ for a composite annular wedge with an apex angle $\beta=120^{\circ}$ and $a=0.4 b$ subjected to a screw dislocation located at $\left(0.6 b, 45^{\circ}\right)$.

$$
\begin{aligned}
\tau_{r z}^{(2)}= & \frac{n \mu_{2} f_{z}}{\pi\left(\mu_{1}+\mu_{2}\right) r}\left[\Gamma^{-}\left((r / d)^{n}, n(\theta-\alpha)\right)+\Gamma^{-}\left((r / d)^{n}, n(\theta+\alpha)\right)-\Gamma^{-}\left(\left(r d / b^{2}\right)^{n}, n(\theta-\alpha)\right)\right. \\
& \left.-\Gamma^{-}\left(\left(r d / b^{2}\right)^{n}, n(\theta+\alpha)\right)\right]
\end{aligned}
$$

\section{A.3. Fixed-fixed-free boundary condition}

$$
\begin{aligned}
w^{(1)}= & \frac{f_{z}}{4 \pi \mu_{1}}\left[\Omega^{-}\left((r / d)^{n}, n(\theta-\alpha)\right)-\Omega^{-}\left((r / d)^{n}, n(\theta+\alpha)\right)-k \Omega^{+}\left((r / d)^{n}, n(\theta-\alpha)\right)\right. \\
& +k \Omega^{+}\left((r / d)^{n}, n(\theta+\alpha)\right)+\Omega^{-}\left(\left(r d / b^{2}\right)^{n}, n(\theta-\alpha)\right)-\Omega^{-}\left(\left(r d / b^{2}\right)^{n}, n(\theta+\alpha)\right) \\
& \left.-k \Omega^{+}\left(\left(r d / b^{2}\right)^{n}, n(\theta-\alpha)\right)+k \Omega^{+}\left(\left(r d / b^{2}\right)^{n}, n(\theta+\alpha)\right)\right]
\end{aligned}
$$



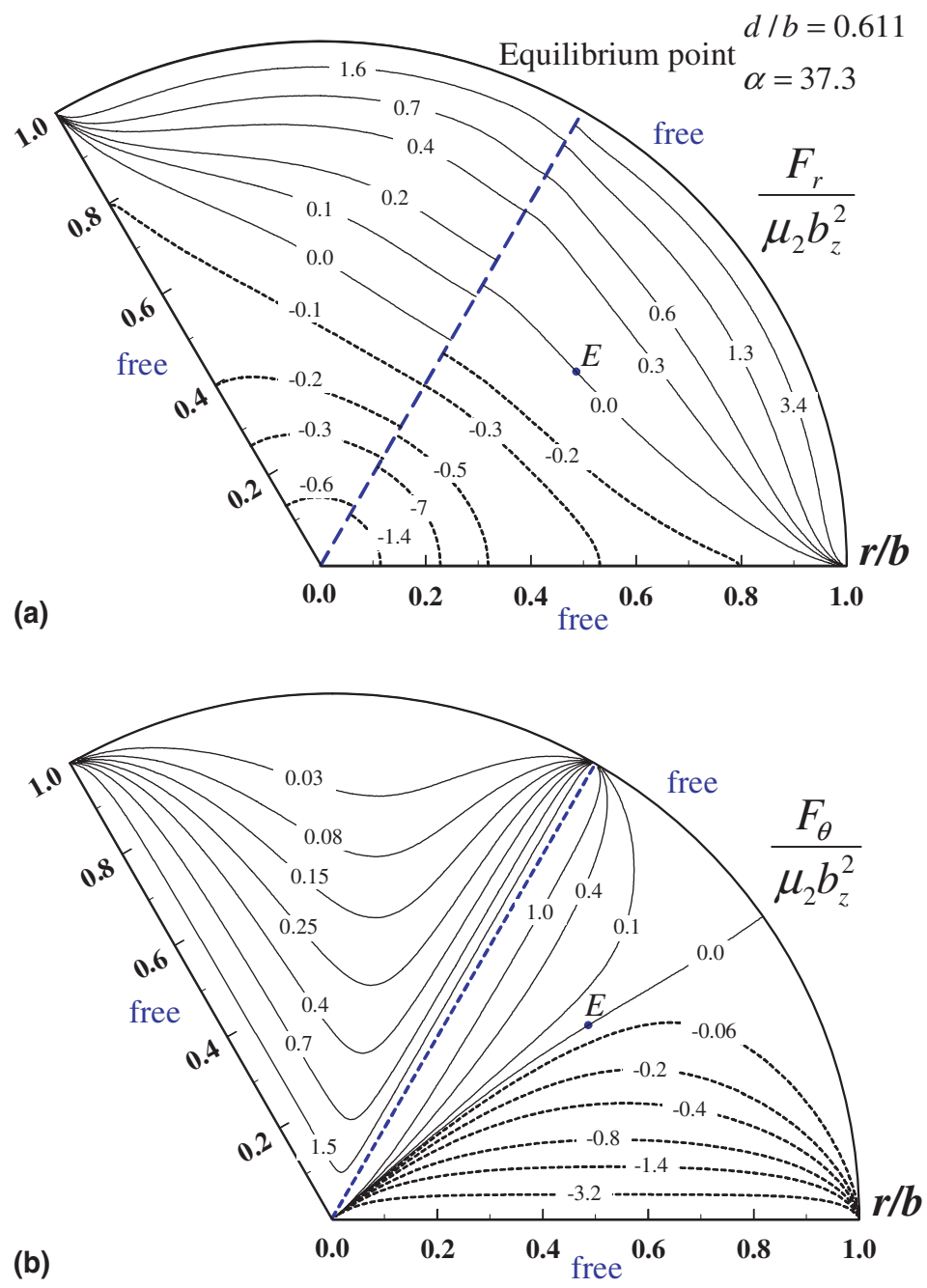

Fig. 20. (a) Image force $F_{r}$ exerted on a screw dislocation in a composite wedge with apex angle $\beta=120^{\circ}$ and finite radius $r=b$. (b) Image force $F_{\theta}$ exerted on a screw dislocation in a composite wedge with apex angle $\beta=120^{\circ}$ and finite radius $r=b$.

$$
\begin{aligned}
\tau_{r z}^{(1)}= & \frac{n f_{z}}{2 \pi r}\left[\Gamma^{-}\left((r / d)^{n}, n(\theta-\alpha)\right)-\Gamma^{-}\left((r / d)^{n}, n(\theta+\alpha)\right)-k \Gamma^{+}\left((r / d)^{n}, n(\theta-\alpha)\right)\right. \\
& +k \Gamma^{+}\left((r / d)^{n}, n(\theta+\alpha)\right)+\Gamma^{-}\left(\left(r d / b^{2}\right)^{n}, n(\theta-\alpha)\right)-\Gamma^{-}\left(\left(r d / b^{2}\right)^{n}, n(\theta+\alpha)\right) \\
& \left.-k \Gamma^{+}\left(\left(r d / b^{2}\right)^{n}, n(\theta-\alpha)\right)+k \Gamma^{+}\left(\left(r d / b^{2}\right)^{n}, n(\theta+\alpha)\right)\right] \\
w^{(2)}= & \frac{f_{z}}{2 \pi\left(\mu_{1}+\mu_{2}\right)}\left[\Omega^{-}\left((r / d)^{n}, n(\theta-\alpha)\right)-\Omega^{-}\left((r / d)^{n}, n(\theta+\alpha)\right)+\Omega^{-}\left(\left(r d / b^{2}\right)^{n}, n(\theta-\alpha)\right)\right. \\
& \left.-\Omega^{-}\left(\left(r d / b^{2}\right)^{n}, n(\theta+\alpha)\right)\right]
\end{aligned}
$$



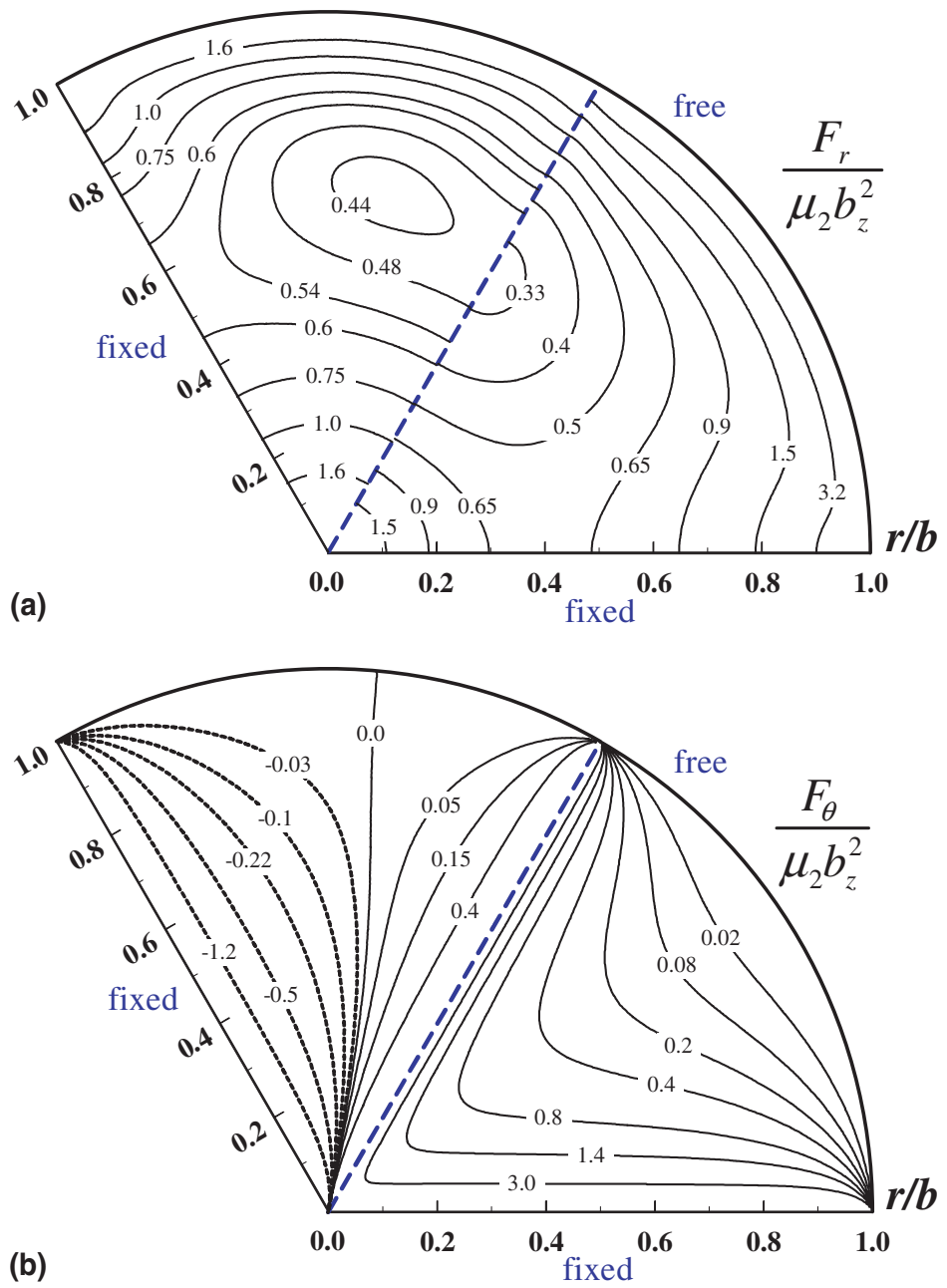

Fig. 21. (a) Image force $F_{r}$ exerted on a screw dislocation in a composite wedge with apex angle $\beta=120^{\circ}$ and finite radius $r=b$. (b) Image force $F_{\theta}$ exerted on a screw dislocation in a composite wedge with apex angle $\beta=120^{\circ}$ and finite radius $r=b$.

$$
\begin{aligned}
\tau_{r z}^{(2)}= & \frac{n \mu_{2} f_{z}}{\pi\left(\mu_{1}+\mu_{2}\right) r}\left[\Gamma^{-}\left((r / d)^{n}, n(\theta-\alpha)\right)-\Gamma^{-}\left((r / d)^{n}, n(\theta+\alpha)\right)+\Gamma^{-}\left(\left(r d / b^{2}\right)^{n}, n(\theta-\alpha)\right)\right. \\
& \left.-\Gamma^{-}\left(\left(r d / b^{2}\right)^{n}, n(\theta+\alpha)\right)\right]
\end{aligned}
$$

\section{A.4. Fixed-fixed-fixed boundary condition}

$$
\begin{aligned}
w^{(1)}= & \frac{f_{z}}{4 \pi \mu_{1}}\left[\Omega^{-}\left((r / d)^{n}, n(\theta-\alpha)\right)-\Omega^{-}\left((r / d)^{n}, n(\theta+\alpha)\right)-k \Omega^{+}\left((r / d)^{n}, n(\theta-\alpha)\right)\right. \\
& +k \Omega^{+}\left((r / d)^{n}, n(\theta+\alpha)\right)-\Omega^{-}\left(\left(r d / b^{2}\right)^{n}, n(\theta-\alpha)\right)+\Omega^{-}\left(\left(r d / b^{2}\right)^{n}, n(\theta+\alpha)\right) \\
& \left.+k \Omega^{+}\left(\left(r d / b^{2}\right)^{n}, n(\theta-\alpha)\right)-k \Omega^{+}\left(\left(r d / b^{2}\right)^{n}, n(\theta+\alpha)\right)\right]
\end{aligned}
$$



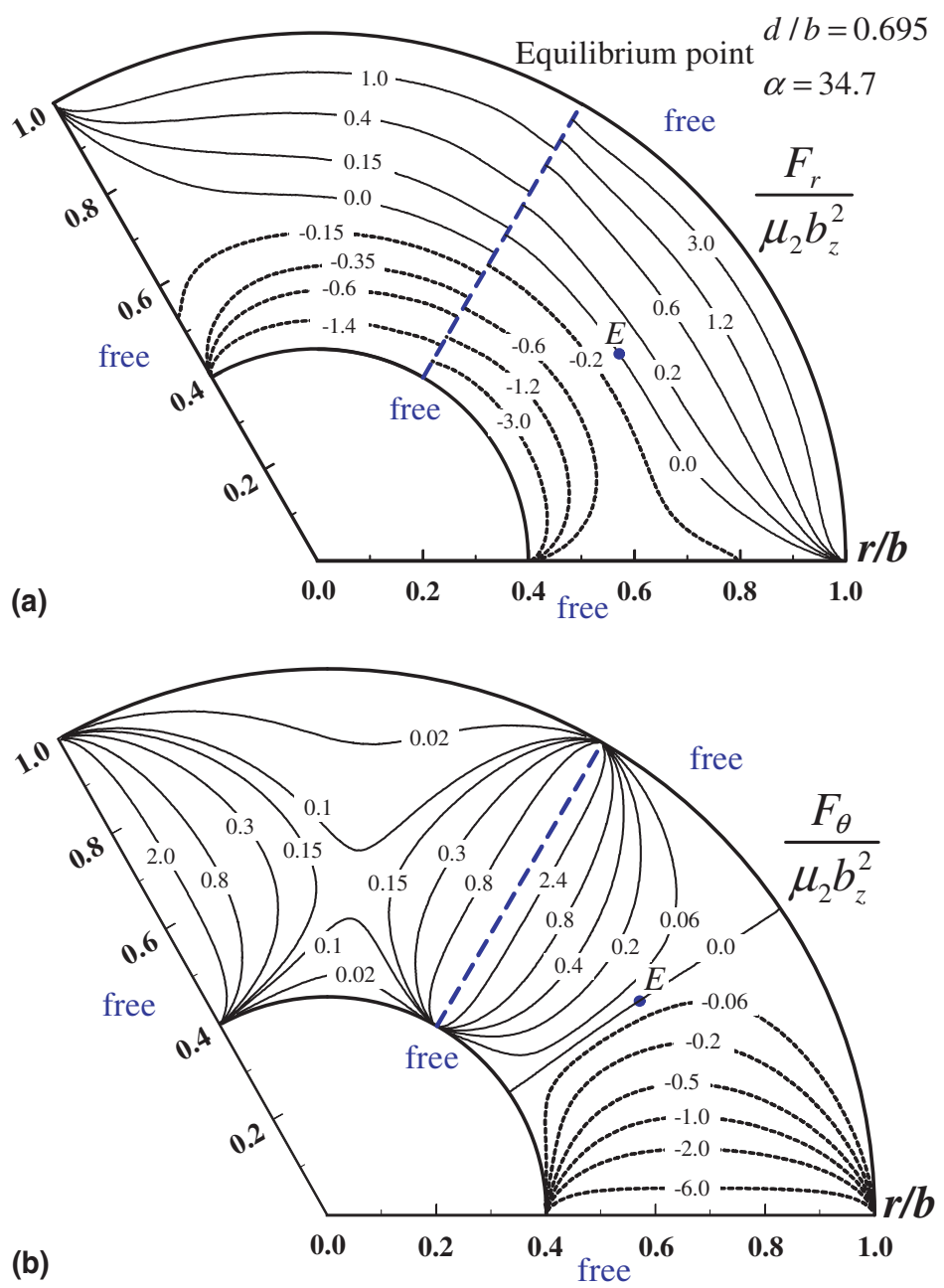

Fig. 22. (a) Image force $F_{r}$ exerted on a screw dislocation in an annular wedge with apex angle $\beta=120^{\circ}$. (b) Image force $F_{\theta}$ exerted on a screw dislocation in an annular wedge with apex angle $\beta=120^{\circ}$.

$$
\begin{aligned}
\tau_{r z}^{(1)}= & \frac{n f_{z}}{2 \pi r}\left[\Gamma^{-}\left((r / d)^{n}, n(\theta-\alpha)\right)-\Gamma^{-}\left((r / d)^{n}, n(\theta+\alpha)\right)-k \Gamma^{+}\left((r / d)^{n}, n(\theta-\alpha)\right)\right. \\
& +k \Gamma^{+}\left((r / d)^{n}, n(\theta+\alpha)\right)-\Gamma^{-}\left(\left(r d / b^{2}\right)^{n}, n(\theta-\alpha)\right)+\Gamma^{-}\left(\left(r d / b^{2}\right)^{n}, n(\theta+\alpha)\right) \\
& \left.+k \Gamma^{+}\left(\left(r d / b^{2}\right)^{n}, n(\theta-\alpha)\right)-k \Gamma^{+}\left(\left(r d / b^{2}\right)^{n}, n(\theta+\alpha)\right)\right] \\
w^{(2)}= & \frac{f_{z}}{2 \pi\left(\mu_{1}+\mu_{2}\right)}\left[\Omega^{-}\left((r / d)^{n}, n(\theta-\alpha)\right)-\Omega^{-}\left((r / d)^{n}, n(\theta+\alpha)\right)-\Omega^{-}\left(\left(r d / b^{2}\right)^{n}, n(\theta-\alpha)\right)\right. \\
& \left.+\Omega^{-}\left(\left(r d / b^{2}\right)^{n}, n(\theta+\alpha)\right)\right] \\
\tau_{r z}^{(2)}= & \frac{n \mu_{2} f_{z}}{\pi\left(\mu_{1}+\mu_{2}\right) r}\left[\Gamma^{-}\left((r / d)^{n}, n(\theta-\alpha)\right)-\Gamma^{-}\left((r / d)^{n}, n(\theta+\alpha)\right)-\Gamma^{-}\left(\left(r d / b^{2}\right)^{n}, n(\theta-\alpha)\right)\right. \\
& \left.+\Gamma^{-}\left(\left(r d / b^{2}\right)^{n}, n(\theta+\alpha)\right)\right]
\end{aligned}
$$


Appendix B. The full-field solutions $w(r, \theta)$ and $\tau_{r z}(r, \theta)$ of composite sharp wedges with finite radius (screw dislocations)

B.1. Free-free-free boundary condition

$$
\begin{aligned}
w^{(1)}= & \frac{-b_{z}}{2 \pi}\left[\Psi^{-}\left((r / d)^{n}, n(\theta-\alpha)\right)-\Psi^{-}\left((r / d)^{n}, n(\theta+\alpha)\right)+k \Psi^{+}\left((r / d)^{n}, n(\theta-\alpha)\right)\right. \\
& -k \Psi^{+}\left((r / d)^{n}, n(\theta+\alpha)\right)-\Psi^{-}\left(\left(r d / b^{2}\right)^{n}, n(\theta-\alpha)\right)+\Psi^{-}\left(\left(r d / b^{2}\right)^{n}, n(\theta+\alpha)\right) \\
& \left.-k \Psi^{+}\left(\left(r d / b^{2}\right)^{n}, n(\theta-\alpha)\right)+k \Psi^{+}\left(\left(r d / b^{2}\right)^{n}, n(\theta+\alpha)\right)\right] \\
\tau_{r z}^{(1)}= & \frac{n \mu_{1} b_{z}}{2 \pi r}\left[\Theta^{-}\left((r / d)^{n}, n(\theta-\alpha)\right)-\Theta^{-}\left((r / d)^{n}, n(\theta+\alpha)\right)-k \Theta^{+}\left((r / d)^{n}, n(\theta-\alpha)\right)\right. \\
& +k \Theta^{+}\left((r / d)^{n}, n(\theta+\alpha)\right)-\Theta^{-}\left(\left(r d / b^{2}\right)^{n}, n(\theta-\alpha)\right)+\Theta^{-}\left(\left(r d / b^{2}\right)^{n}, n(\theta+\alpha)\right) \\
& \left.+k \Theta^{+}\left(\left(r d / b^{2}\right)^{n}, n(\theta-\alpha)\right)-k \Theta^{+}\left(\left(r d / b^{2}\right)^{n}, n(\theta+\alpha)\right)\right] \\
w^{(2)}= & \frac{-\mu_{1} b_{z}}{\pi\left(\mu_{1}+\mu_{2}\right)}\left[\Psi^{-}\left((r / d)^{n}, n(\theta-\alpha)\right)-\Psi^{-}\left((r / d)^{n}, n(\theta+\alpha)\right)-\Psi^{-}\left(\left(r d / b^{2}\right)^{n}, n(\theta-\alpha)\right)\right. \\
& \left.+\Psi^{-}\left(\left(r d / b^{2}\right)^{n}, n(\theta+\alpha)\right)\right] \\
\tau_{r z}^{(2)}= & \frac{n \mu_{1} \mu_{2} b_{z}}{\pi\left(\mu_{1}+\mu_{2}\right) r}\left[\Theta^{-}\left((r / d)^{n}, n(\theta-\alpha)\right)-\Theta^{-}\left((r / d)^{n}, n(\theta+\alpha)\right)-\Theta^{-}\left(\left(r d / b^{2}\right)^{n}, n(\theta-\alpha)\right)\right. \\
& \left.+\Theta^{-}\left(\left(r d / b^{2}\right)^{n}, n(\theta+\alpha)\right)\right]
\end{aligned}
$$

B.2. Fixed-fixed-free boundary condition

$$
\begin{aligned}
w^{(1)}= & \frac{-b_{z}}{2 \pi}\left[\Psi^{-}\left((r / d)^{n}, n(\theta-\alpha)\right)+\Psi^{-}\left((r / d)^{n}, n(\theta+\alpha)\right)-k \Psi^{+}\left((r / d)^{n}, n(\theta-\alpha)\right)\right. \\
& -k \Psi^{+}\left((r / d)^{n}, n(\theta+\alpha)\right)-\Psi^{-}\left(\left(r d / b^{2}\right)^{n}, n(\theta-\alpha)\right)-\Psi^{-}\left(\left(r d / b^{2}\right)^{n}, n(\theta+\alpha)\right) \\
& \left.+k \Psi^{+}\left(\left(r d / b^{2}\right)^{n}, n(\theta-\alpha)\right)+k \Psi^{+}\left(\left(r d / b^{2}\right)^{n}, n(\theta+\alpha)\right)\right] \\
\tau_{r z}^{(1)}= & \frac{n \mu_{1} b_{z}}{2 \pi r}\left[\Theta^{-}\left((r / d)^{n}, n(\theta-\alpha)\right)+\Theta^{-}\left((r / d)^{n}, n(\theta+\alpha)\right)+k \Theta^{+}\left((r / d)^{n}, n(\theta-\alpha)\right)\right. \\
& +k \Theta^{+}\left((r / d)^{n}, n(\theta+\alpha)\right)-\Theta^{-}\left(\left(r d / b^{2}\right)^{n}, n(\theta-\alpha)\right)-\Theta^{-}\left(\left(r d / b^{2}\right)^{n}, n(\theta+\alpha)\right) \\
& \left.-k \Theta^{+}\left(\left(r d / b^{2}\right)^{n}, n(\theta-\alpha)\right)-k \Theta^{+}\left(\left(r d / b^{2}\right)^{n}, n(\theta+\alpha)\right)\right] \\
w^{(2)}= & \frac{-\mu_{1} b_{z}}{\pi\left(\mu_{1}+\mu_{2}\right)}\left[\Psi^{-}\left((r / d)^{n}, n(\theta-\alpha)\right)+\Psi^{-}\left((r / d)^{n}, n(\theta+\alpha)\right)-\Psi^{-}\left(\left(r d / b^{2}\right)^{n}, n(\theta-\alpha)\right)\right. \\
& \left.-\Psi^{-}\left(\left(r d / b^{2}\right)^{n}, n(\theta+\alpha)\right)\right] \\
\tau_{r z}^{(2)}= & \frac{n \mu_{1} \mu_{2} b_{z}}{\pi\left(\mu_{1}+\mu_{2}\right) r}\left[\Theta^{-}\left((r / d)^{n}, n(\theta-\alpha)\right)+\Theta^{-}\left((r / d)^{n}, n(\theta+\alpha)\right)-\Theta^{-}\left(\left(r d / b^{2}\right)^{n}, n(\theta-\alpha)\right)\right. \\
& \left.-\Theta^{-}\left(\left(r d / b^{2}\right)^{n}, n(\theta+\alpha)\right)\right]
\end{aligned}
$$




\section{Appendix $\mathrm{C}$. The full-field solutions $w(r, \theta)$ and $\tau_{r z}(r, \theta)$ of composite annular wedge (concentrated loads)}

C.1. Free-free-free-free boundary condition

$$
\begin{aligned}
& w^{(1)}=\frac{f_{z}}{4 \pi \mu_{1}} \sum_{\ell=0}^{\infty} \sum_{j=1}^{4} \sum_{m=1}^{2}(-1)^{m+1}\left[\Omega^{-}\left(r_{j}, \phi^{-}\right)+\Omega^{-}\left(r_{j}, \phi^{+}\right)+k \Omega^{+}\left(r_{j}, \phi^{-}\right)+k \Omega^{+}\left(r_{j}, \phi^{+}\right)\right] \\
& \tau_{r z}^{(1)}=\frac{n f_{z}}{2 \pi r} \sum_{\ell=0}^{\infty} \sum_{j=1}^{4} \sum_{m=1}^{2}(-1)^{m+1}\left[\Gamma^{-}\left(r_{j}, \phi^{-}\right)+\Gamma^{-}\left(r_{j}, \phi^{+}\right)+k \Gamma^{+}\left(r_{j}, \phi^{-}\right)+k \Gamma^{+}\left(r_{j}, \phi^{+}\right)\right] \\
& w^{(2)}=\frac{f_{z}}{2 \pi\left(\mu_{1}+\mu_{2}\right)} \sum_{\ell=0}^{\infty} \sum_{j=1}^{4} \sum_{m=1}^{2}(-1)^{m+1}\left[\Omega^{-}\left(r_{j}, \phi^{-}\right)+\Omega^{-}\left(r_{j}, \phi^{+}\right)\right] \\
& \tau_{r z}^{(2)}=\frac{n \mu_{2} f_{z}}{\pi\left(\mu_{1}+\mu_{2}\right) r} \sum_{\ell=0}^{\infty} \sum_{j=1}^{4} \sum_{m=1}^{2}(-1)^{m+1}\left[\Gamma^{-}\left(r_{j}, \phi^{-}\right)+\Gamma^{-}\left(r_{j}, \phi^{+}\right)\right]
\end{aligned}
$$

\section{C.2. Fixed-fixed-free-free boundary condition}

$$
\begin{aligned}
w^{(1)} & =\frac{f_{z}}{4 \pi \mu_{1}} \sum_{\ell=0}^{\infty} \sum_{j=1}^{4}\left[\Omega^{-}\left(r_{j}, \phi^{-}\right)-\Omega^{-}\left(r_{j}, \phi^{+}\right)-k \Omega^{+}\left(r_{j}, \phi^{-}\right)+k \Omega^{+}\left(r_{j}, \phi^{+}\right)\right] \\
\tau_{r z}^{(1)} & =\frac{n f_{z}}{2 \pi r} \sum_{\ell=0}^{\infty} \sum_{j=1}^{4}\left[\Gamma^{-}\left(r_{j}, \phi^{-}\right)-\Gamma^{-}\left(r_{j}, \phi^{+}\right)-k \Gamma^{+}\left(r_{j}, \phi^{-}\right)+k \Gamma^{+}\left(r_{j}, \phi^{+}\right)\right] \\
w^{(2)} & =\frac{f_{z}}{2 \pi\left(\mu_{1}+\mu_{2}\right)} \sum_{\ell=0}^{\infty} \sum_{j=1}^{4}\left[\Omega^{-}\left(r_{j}, \phi^{-}\right)-\Omega^{-}\left(r_{j}, \phi^{+}\right)\right] \\
\tau_{r z}^{(2)} & =\frac{n \mu_{2} f_{z}}{\pi\left(\mu_{1}+\mu_{2}\right) r} \sum_{\ell=0}^{\infty} \sum_{j=1}^{4}\left[\Gamma^{-}\left(r_{j}, \phi^{-}\right)-\Gamma^{-}\left(r_{j}, \phi^{+}\right)\right]
\end{aligned}
$$

C.3. Free-free-fixed-free boundary condition

$$
\begin{aligned}
& w^{(1)}=\frac{f_{z}}{4 \pi \mu_{1}} \sum_{\ell=0}^{\infty} \sum_{j=1}^{4}(-1)^{\ell+j-1}\left[\Omega^{-}\left(r_{j}, \phi^{-}\right)+\Omega^{-}\left(r_{j}, \phi^{+}\right)+k \Omega^{+}\left(r_{j}, \phi^{-}\right)+k \Omega^{+}\left(r_{j}, \phi^{+}\right)\right] \\
& \tau_{r z}^{(1)}=\frac{n f_{z}}{2 \pi r} \sum_{\ell=0}^{\infty} \sum_{j=1}^{4}(-1)^{\ell+j-1}\left[\Gamma^{-}\left(r_{j}, \phi^{-}\right)+\Gamma^{-}\left(r_{j}, \phi^{+}\right)+k \Gamma^{+}\left(r_{j}, \phi^{-}\right)+k \Gamma^{+}\left(r_{j}, \phi^{+}\right)\right] \\
& w^{(2)}=\frac{f_{z}}{2 \pi\left(\mu_{1}+\mu_{2}\right)} \sum_{\ell=0}^{\infty} \sum_{j=1}^{4}(-1)^{\ell+j-1}\left[\Omega^{-}\left(r_{j}, \phi^{-}\right)+\Omega^{-}\left(r_{j}, \phi^{+}\right)\right]
\end{aligned}
$$




$$
\tau_{r z}^{(2)}=\frac{n \mu_{2} f_{z}}{\pi\left(\mu_{1}+\mu_{2}\right) r} \sum_{\ell=0}^{\infty} \sum_{j=1}^{4}(-1)^{\ell+j-1}\left[\Gamma^{-}\left(r_{j}, \phi^{-}\right)+\Gamma^{-}\left(r_{j}, \phi^{+}\right)\right]
$$

\section{Appendix D. The full-field solutions $w(r, \theta)$ and $\tau_{r z}(r, \theta)$ of composite annular wedge (screw dislocations)}

D.1. Free-free-free-free boundary condition

$$
\begin{aligned}
& w^{(1)}=\frac{b_{z}}{2 \pi} \sum_{\ell=0}^{\infty} \sum_{j=1}^{4}(-1)^{j}\left[\Psi^{-}\left(r_{j}, \phi^{-}\right)-\Psi^{-}\left(r_{j}, \phi^{+}\right)+k \Psi^{+}\left(r_{j}, \phi^{-}\right)-k \Psi^{+}\left(r_{j}, \phi^{+}\right)\right] \\
& \tau_{r z}^{(1)}=\frac{n \mu_{1} b_{z}}{2 \pi r} \sum_{\ell=0}^{\infty} \sum_{j=1}^{4}(-1)^{j+1}\left[\Theta^{-}\left(r_{j}, \phi^{-}\right)-\Theta^{-}\left(r_{j}, \phi^{+}\right)-k \Theta^{+}\left(r_{j}, \phi^{-}\right)+k \Theta^{+}\left(r_{j}, \phi^{+}\right)\right] \\
& w^{(2)}=\frac{\mu_{1} b_{z}}{\pi\left(\mu_{1}+\mu_{2}\right)} \sum_{\ell=0}^{\infty} \sum_{j=1}^{4}(-1)^{j}\left[\Psi^{-}\left(r_{j}, \phi^{-}\right)-\Psi^{-}\left(r_{j}, \phi^{+}\right)\right] \\
& \tau_{r z}^{(2)}=\frac{n \mu_{1} \mu_{2} b_{z}}{\pi\left(\mu_{1}+\mu_{2}\right) r} \sum_{\ell=0}^{\infty} \sum_{j=1}^{4}(-1)^{j+1}\left[\Theta^{-}\left(r_{j}, \phi^{-}\right)-\Theta^{-}\left(r_{j}, \phi^{+}\right)\right]
\end{aligned}
$$

\section{D.2. Fixed-fixed-free-fixed boundary condition}

$$
\begin{aligned}
& w^{(1)}=\frac{b_{z}}{2 \pi} \sum_{\ell=0}^{\infty} \sum_{j=1}^{4}(-1)^{\ell+j}\left[\Psi^{-}\left(r_{j}, \phi^{-}\right)+\Psi^{-}\left(r_{j}, \phi^{+}\right)-k \Psi^{+}\left(r_{j}, \phi^{-}\right)-k \Psi^{+}\left(r_{j}, \phi^{+}\right)\right] \\
& \tau_{r z}^{(1)}=\frac{-n \mu_{1} b_{z}}{2 \pi r} \sum_{\ell=0}^{\infty} \sum_{j=1}^{4}(-1)^{\ell+j}\left[\Theta^{-}\left(r_{j}, \phi^{-}\right)+\Theta^{-}\left(r_{j}, \phi^{+}\right)+k \Theta^{+}\left(r_{j}, \phi^{-}\right)+k \Theta^{+}\left(r_{j}, \phi^{+}\right)\right] \\
& w^{(2)}=\frac{\mu_{1} b_{z}}{\pi\left(\mu_{1}+\mu_{2}\right)} \sum_{\ell=0}^{\infty} \sum_{j=1}^{4}(-1)^{\ell+j}\left[\Psi^{-}\left(r_{j}, \phi^{-}\right)+\Psi^{-}\left(r_{j}, \phi^{+}\right)\right] \\
& \tau_{r z}^{(2)}=\frac{-n \mu_{1} \mu_{2} b_{z}}{\pi\left(\mu_{1}+\mu_{2}\right) r} \sum_{\ell=0}^{\infty} \sum_{j=1}^{4}(-1)^{\ell+j}\left[\Theta^{-}\left(r_{j}, \phi^{-}\right)+\Theta^{-}\left(r_{j}, \phi^{+}\right)\right]
\end{aligned}
$$

\section{References}

Bogy, D.B., 1971. Two edge-bonded elastic wedges of different materials and wedge under surface tractions. Journal of Applied Mechanics 35, 460-466.

Bogy, D.B., 1972. The plane solution for anisotropic elastic wedge under normal and shear loading. Journal of Applied Mechanics 39, $1103-1109$.

Chou, Y.T., 1965. Screw dislocations near a wedge-shaped boundary. Acta Metallurgica 13, 1131-1134.

Chou, Y.T., 1966. Screw dislocations in and near lamellar inclusions. Physica Status Solidi 17, 509-516. 
Chu, S.N.G., 1982. Screw dislocation in a two-phase isotropic thin film. Journal of Applied Physics 53, 3019-3023.

He, M.Y., Hutchinson, J.W., 1989a. Kinking of a crack out of an interface. Journal of Applied Mechanics 56, $270-278$.

He, M.Y., Hutchinson, J.W., 1989b. Crack deflection at an interface between dissimilar elastic material. International Journal of Solids and Structures 25, 1053-1067.

Kargarnovin, M.H., 2000. Analysis of a dissimilar finite wedge under antiplane deformation. Mechanics Research Communications 27, 109-116.

Kargarnovin, M.H., Shahani, A.R., Fariborz, S.F., 1997. Analysis of an isotropic finite wedge under antiplane deformation. International Journal of Solids and Structures 34, 113-128.

Lin, L.S., Chou, Y.T., 1975. Screw dislocations in a three-phase anisotropic medium. International Journal of Engineering Science 13, 317-325.

Lin, R.L., Ma, C.C., 2000. Antiplane deformation for anisotropic multilayered media by using the coordinate transform method. Journal of Applied Mechanics 67, 597-605.

Lin, R.L., Ma, C.C., 2003. Analytic full-field solutions of screw dislocation in finite angular wedges. The Chinese Journal of Mechanics (Series A) 19, 83-98.

Ma, C.C., Hour, B.L., 1989. Analysis of dissimilar anisotropic wedges subjected to antiplane shear deformation. International Journal of Solids and Structures 25, 1295-1309.

Shahani, A.R., 1999. Analysis of an anisotropic finite wedge under antiplane deformation. Journal of Elasticity 56, 17-32.

Ting, T.C.T., 1984. The wedge subjected to tractions: a paradox re-examined. Journal of Elasticity 14, 235-247.

Ting, T.C.T., 1985. Elastic wedge subjected to antiplane shear traction-A paradox explained. The Quarterly Journal of Mechanics and Applied Mathematics 38, 245-255.

Tranter, C.J., 1948. The use of the Mellin transform in finding the stress distribution in an infinite wedge. The Quarterly Journal of Mechanics and Applied Mathematics 1, 125-130.

Wang, Z.Y., Zhang, H.T., Chou, Y.T., 1986. Stress singularity at the tip of a rigid line inhomogeneity under antiplane shear loading. Journal of Applied Mechanics 53, 459-461.

Williams, M.L., 1952. Stress singularities resulting from various boundary condition in angular corners of plates in extension. Journal of Applied Mechanics 19, 526-528.

Zhang, T.Y., Tong, P., Ouyang, H., Lee, S., 1995. Internation of an edge dislocation with a wedge crack. Journal of Applied Physics 78, 4873-8233. 